\title{
SPECTRAL ANALYSIS AND INTERPRETATION OF THE $\gamma$-RAY EMISSION FROM THE STARBURST GALAXY NGC 253*
}

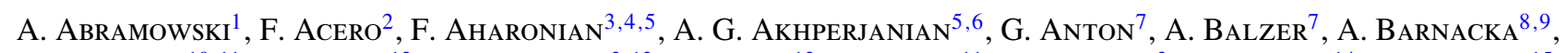

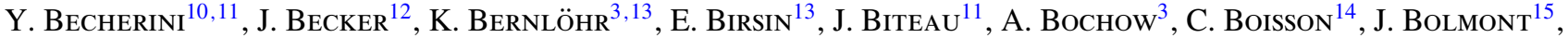
P. Bordas ${ }^{16}$, J. Brucker ${ }^{7}$, F. Brun ${ }^{11}$, P. Brun ${ }^{9}$, T. Bulik ${ }^{17}$, I. Büsching ${ }^{12,18}$, S. Carrigan ${ }^{3}$, S. Casanova ${ }^{3,18}$, M. Cerruti ${ }^{14}$, P. M. Chadwick ${ }^{19}$, A. Charbonnier ${ }^{15}$, R. C. G. Chaves ${ }^{3,9}$, A. Cheesebrough ${ }^{19}$, G. Cologna ${ }^{20}$, J. Conrad ${ }^{21}$, C. Couturier ${ }^{15}$, M. Dalton ${ }^{13}$, M. K. Daniel ${ }^{19}$, I. D. Davids ${ }^{22}$, B. Degraname ${ }^{11}$, C. Deil ${ }^{3}$, H. J. Dickinson ${ }^{21}$, A. Djannati-Atä̈ ${ }^{10}$, W. Domainko ${ }^{3}$, L.O’C. Drury 4 , G. Dubus ${ }^{23}$, K. Dutson ${ }^{24}$, J. Dyks ${ }^{8}$, M. Dyrda ${ }^{25}$, K. Egberts ${ }^{26}$, P. Eger ${ }^{7}$, P. Espigat ${ }^{10}$, L. Fallon ${ }^{4}$, S. Fegan ${ }^{11}$, F. Feinstein ${ }^{2}$, M. V. Fernandes ${ }^{1}$, A. Fiasson ${ }^{27}$, G. Fontaine ${ }^{11}$, A. Förster ${ }^{3}$, M. Füßling ${ }^{13}$, M. Gajdus ${ }^{13}$, Y. A. Gallant ${ }^{2}$, T. Garrigoux ${ }^{15}$, H. Gast ${ }^{3}$, L. Gérard ${ }^{10}$, B. Giebels ${ }^{11}$, J. F. Glicenstein ${ }^{9}$, B. GlÜCK ${ }^{7}$,

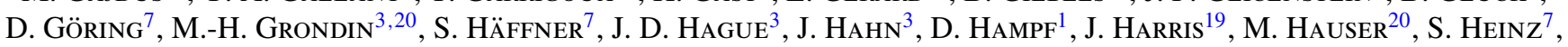
G. HeinzelmanN ${ }^{1}$, G. Henri ${ }^{23}$, G. HermanN ${ }^{3}$, A. Hillert ${ }^{3}$, J. A. Hinton ${ }^{24}$, W. HofmanN ${ }^{3}$, P. Hofverberg ${ }^{3}$, M. Holler ${ }^{7}$,

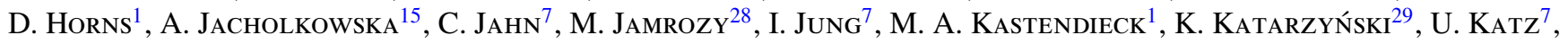
S. KaufmanN ${ }^{20}$, B. Khélifi ${ }^{11}$, D. KlochKov ${ }^{16}$, W. KLuŹNiaK ${ }^{8}$, T. KNeISKe ${ }^{1}$, Nu. Komin ${ }^{27}$, K. KosaCK ${ }^{9}$, R. KossaKowsKi ${ }^{27}$, F. KRAYZEL ${ }^{27}$, H. LAFFON ${ }^{11}$, G. LAMANNA ${ }^{27}$, J.-P. Lenain ${ }^{20}$, D. LenNARZ ${ }^{3}$, T. LOHSE ${ }^{13}$, A. LOPATIN ${ }^{7}$, C.-C. LU ${ }^{3}$, V. MARANDON ${ }^{3}$, A. Marcowith ${ }^{2}$, J. Masbou ${ }^{27}$, G. Maurin ${ }^{27}$, N. Maxted ${ }^{30}$, M. Mayer ${ }^{7}$, T. J. L. McComb ${ }^{19}$, M. C. Medina ${ }^{9}$, J. Méhault ${ }^{2}$, R. Moderski ${ }^{8}$, M. Mohamed ${ }^{20}$, E. Moulin ${ }^{9}$, C. L. NaumanN ${ }^{15}$, M. Naumann-Godo ${ }^{9}$, M. De Naurois ${ }^{11}$, D. Nedbat ${ }^{31,33}$, D. Nekrassov ${ }^{3}$, N. NGuYen ${ }^{1}$, B. Nicholas ${ }^{30}$, J. Niemiec ${ }^{25}$, S. J. Nolan ${ }^{19}$, S. OhM ${ }^{3,24,32}$, E. De OÑa Wilhelmi ${ }^{3}$, B. Opitz ${ }^{1}$, M. Ostrowski ${ }^{28}$, I. Oya ${ }^{13}$, M. Panter ${ }^{3}$, M. Paz Arribas ${ }^{13}$, N. W. Pekeur ${ }^{18}$, G. Pelletier ${ }^{23}$, J. Perez ${ }^{26}$, P.-O. Petruccir ${ }^{23}$, B. Peyaud ${ }^{9}$, S. Pita ${ }^{10}$, G. PÜhlhofer ${ }^{16}$, M. Punch ${ }^{10}$, A. Quirrenbach ${ }^{20}$, M. Raue ${ }^{1}$, A. Reimer ${ }^{26}$, O. Reimer ${ }^{26}$, M. Renaud $^{2}$,

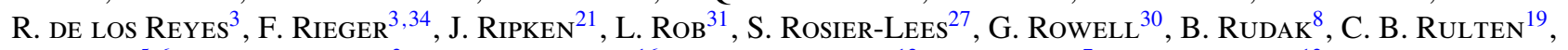
V. Sahakian ${ }^{5,6}$, D. A. Sanchez ${ }^{3}$, A. Santangelo ${ }^{16}$, R. Schlickeiser ${ }^{12}$, A. Schulz ${ }^{7}$, U. Schwanke ${ }^{13}$, S. Schwarzburg ${ }^{16}$,

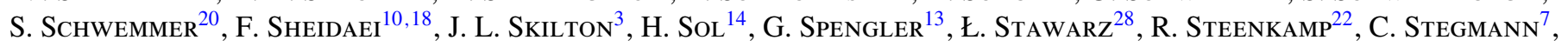

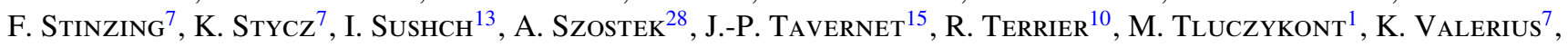
C. van Eldik ${ }^{3,7}$, G. Vasileiadis ${ }^{2}$, C. Venter ${ }^{18}$, A. Viana ${ }^{9}$, P. Vincent ${ }^{15}$, H. J. VölK ${ }^{3}$, F. Volpe ${ }^{3}$, S. Vorobiov ${ }^{2}$, M. Vorster ${ }^{18}$, S. J. WAGner ${ }^{20}$, M. WArd ${ }^{19}$, R. White ${ }^{24}$, A. WierzcholsKa ${ }^{28}$, M. Zacharias ${ }^{12}$, A. ZajCZyK ${ }^{2,8}$, A. A. ZDZIARSKI ${ }^{8}$, A. ZeCH ${ }^{14}$, AND H.-S. ZeChLIN ${ }^{1}$

(H.E.S.S. COLLABORATION)
${ }^{1}$ Institut für Experimentalphysik, Universität Hamburg, Luruper Chaussee 149, D-22761 Hamburg, Germany

${ }^{2}$ Laboratoire Univers et Particules de Montpellier, Université Montpellier 2, CNRS/IN2P3, CC 72, Place Eugène Bataillon, F-34095 Montpellier Cedex 5, France

${ }^{3}$ Max-Planck-Institut für Kernphysik, P.O. Box 103980, D-69029 Heidelberg, Germany; stefan.ohm@1e.ac.uk

${ }^{4}$ Dublin Institute for Advanced Studies, 31 Fitzwilliam Place, Dublin 2, Ireland

${ }^{5}$ National Academy of Sciences of the Republic of Armenia, Yerevan, Armenia

${ }^{6}$ Yerevan Physics Institute, 2 Alikhanian Brothers St., 375036 Yerevan, Armenia

${ }^{7}$ Physikalisches Institut, Universität Erlangen-Nürnberg, Erwin-Rommel-Str. 1, D-91058 Erlangen, Germany

${ }^{8}$ Nicolaus Copernicus Astronomical Center, ul. Bartycka 18, 00-716 Warsaw, Poland ${ }^{9}$ CEA Saclay, DSM/IRFU, F-91191 Gif-Sur-Yvette Cedex, France

${ }^{10}$ APC, AstroParticule et Cosmologie, Université Paris Diderot, CNRS/IN2P3, CEA/lrfu, Observatoire de Paris, Sorbonne Paris Cité, 10, rue Alice Domon et Léonie Duquet, F-75205 Paris Cedex 13, France

${ }^{11}$ Laboratoire Leprince-Ringuet, Ecole Polytechnique, CNRS/IN2P3, F-91128 Palaiseau, France; denauroi@in2p3.fr

${ }^{12}$ Institut für Theoretische Physik, Lehrstuhl IV: Weltraum und Astrophysik, Ruhr-Universität Bochum, D-44780 Bochum, Germany

${ }^{13}$ Institut für Physik, Humboldt-Universität zu Berlin, Newtonstr. 15, D-12489 Berlin, Germany

${ }^{14}$ LUTH, Observatoire de Paris, CNRS, Université Paris Diderot, 5 Place Jules Janssen, F-92190 Meudon, France

${ }^{15}$ LPNHE, Université Pierre et Marie Curie Paris 6, Université Denis Diderot Paris 7, CNRS/IN2P3, 4 Place Jussieu, F-75252, Paris Cedex 5, France

${ }^{16}$ Institut für Astronomie und Astrophysik, Universität Tübingen, Sand 1, D-72076 Tübingen, Germany

${ }^{17}$ Astronomical Observatory, The University of Warsaw, Al. Ujazdowskie 4, 00-478 Warsaw, Poland

${ }^{18}$ Unit for Space Physics, North-West University, Potchefstroom 2520, South Africa

${ }_{19}$ Department of Physics, University of Durham, South Road, Durham DH1 3LE, UK

${ }^{20}$ Landessternwarte, Universität Heidelberg, Königstuhl, D-69117 Heidelberg, Germany

${ }^{21}$ Department of Physics, Oskar Klein Centre, Stockholm University, Albanova University Center, SE-10691 Stockholm, Sweden

22 Department of Physics, University of Namibia, Private Bag 13301, Windhoek, Namibia

${ }^{23}$ Laboratoire d'Astrophysique de Grenoble, INSU/CNRS, Université Joseph Fourier, BP 53, F-38041 Grenoble Cedex 9, France

${ }^{24}$ Department of Physics and Astronomy, The University of Leicester, University Road, Leicester LE1 7RH, UK

${ }^{25}$ Instytut Fizyki Jądrowej PAN, ul. Radzikowskiego 152, 31-342 Kraków, Poland

${ }^{26}$ Institut für Astro- und Teilchenphysik, Leopold-Franzens-Universität Innsbruck, A-6020 Innsbruck, Austria

${ }^{27}$ Laboratoire d'Annecy-le-Vieux de Physique des Particules, Université de Savoie, CNRS/IN2P3, F-74941 Annecy-le-Vieux, France

${ }^{28}$ Obserwatorium Astronomiczne, Uniwersytet Jagielloński, ul. Orla 171, 30-244 Kraków, Poland

29 Toruń Centre for Astronomy, Nicolaus Copernicus University, ul. Gagarina 11, 87-100 Toruń, Poland

${ }^{30}$ School of Chemistry and Physics, University of Adelaide, Adelaide 5005, Australia

${ }^{31}$ Faculty of Mathematics and Physics, Institute of Particle and Nuclear Physics, Charles University, V Holešovičkách 2, 18000 Prague 8, Czech Republic

${ }^{32}$ School of Physics and Astronomy, University of Leeds, Leeds LS2 9JT, UK

Received 2012 May 24; accepted 2012 August 17; published 2012 September 13 


\begin{abstract}
Very high energy (VHE; $E \geqslant 100 \mathrm{GeV}$ ) and high-energy (HE; $100 \mathrm{MeV} \leqslant E \leqslant 100 \mathrm{GeV}$ ) data from $\gamma$-ray observations performed with the H.E.S.S. telescope array and the Fermi-LAT instrument, respectively, are analyzed in order to investigate the non-thermal processes in the starburst galaxy NGC 253. The VHE $\gamma$-ray data can be described by a power law in energy with differential photon index $\Gamma=2.14 \pm 0.18_{\text {stat }} \pm 0.30_{\text {sys }}$ and differential flux normalization at $1 \mathrm{TeV}$ of $F_{0}=\left(9.6 \pm 1.5_{\text {stat }}(+5.7,-2.9)_{\text {sys }}\right) \times 10^{-14} \mathrm{TeV}^{-1} \mathrm{~cm}^{-2} \mathrm{~s}^{-1}$. A power-law fit to the differential HE $\gamma$-ray spectrum reveals a photon index of $\Gamma=2.24 \pm 0.14_{\text {stat }} \pm 0.03_{\text {sys }}$ and an integral flux between $200 \mathrm{MeV}$ and $200 \mathrm{GeV}$ of $F(0.2-200 \mathrm{GeV})=\left(4.9 \pm 1.0_{\text {stat }} \pm 0.3_{\text {sys }}\right) \times 10^{-9} \mathrm{~cm}^{-2} \mathrm{~s}^{-1}$. No evidence for a spectral break or turnover is found over the dynamic range of both the LAT instrument and the H.E.S.S. experiment: a combined fit of a power law to the HE and VHE $\gamma$-ray data results in a differential photon index $\Gamma=2.34 \pm 0.03$ with a $p$-value of $30 \%$. The $\gamma$-ray observations indicate that at least about $20 \%$ of the energy of the cosmic rays (CRs) capable of producing hadronic interactions is channeled into pion production. The smooth alignment between the spectra in the HE and VHE $\gamma$-ray domain suggests that the same transport processes dominate in the entire energy range. Advection is most likely responsible for charged particle removal from the starburst nucleus from GeV to multiple $\mathrm{TeV}$ energies. In a hadronic scenario for the $\gamma$-ray production, the single overall power-law spectrum observed would therefore correspond to the mean energy spectrum produced by the ensemble of CR sources in the starburst region.
\end{abstract}

Key words: convection - diffusion - galaxies: individual (NGC 253) - galaxies: starburst - gamma rays: galaxies - radiation mechanisms: non-thermal

Online-only material: color figures

\section{INTRODUCTION}

Starburst galaxies are galaxies that undergo an epoch of star formation in a very localized region (the starburst region) at a rate that is enhanced in comparison to other, so-called late-type galaxies such as the Milky Way. It is believed that this starburst activity is triggered by either galaxy mergers, a close fly-by of galaxies, or Galactic bar instabilities, where the dynamical equilibrium of the interstellar gas gets disturbed. This leads to the formation of regions of very high density gas, usually at the center of the galaxy, and subsequently to star formation and a strongly increased supernova ( $\mathrm{SN}$ ) explosion rate. SN remnant shocks are widely believed to be acceleration sites of cosmic rays (CRs). This is one reason why starburst regions might have a high CR density. Given the high density of target material that is available for $\mathrm{p}-\mathrm{p}$ interactions and the production of $\pi^{0} \mathrm{~s}$, the starburst nucleus is in addition a promising source of high-energy ( $\mathrm{HE} ; 100 \mathrm{MeV} \leqslant E \leqslant 100 \mathrm{GeV}$ ) and very high energy (VHE; $E \geqslant 100 \mathrm{GeV}) \gamma$-rays. From energetic electrons also bremsstrahlung and inverse Compton $\gamma$-rays are expected. These electrons may be either directly accelerated by the same processes as the nuclear particles or generated in the decays of charged pions from hadronic collisions. They might, however, also be produced in different sources, like in pulsar wind nebulae. Starburst galaxies have been predicted early on to be detectable by present $\gamma$-ray instruments (e.g., Völk et al. 1989, 1996; Akyuz et al. 1991; Paglione et al. 1996).

The spiral galaxy NGC 253 is the closest object in the southern sky that belongs to the class of starburst galaxies. Its distance is measured as 2.6-3.9 Mpc using different distance estimation techniques (Davidge et al. 1991; Karachentsev et al. 2003; Rekola et al. 2005). The reference distance is $d=2.6 \mathrm{Mpc}$ (Davidge et al. 1991) since this value is used most widely in

\footnotetext{
* We dedicate this paper to the memory of our colleague Dalibor Nedbal, who died on 2012 May 15 at the age of 31. Dalibor was universally liked and respected as a scientist and colleague and will be greatly missed. 33 Deceased.

${ }^{34}$ European Associated Laboratory for Gamma-Ray Astronomy, jointly supported by CNRS and MPG.
}

the literature to determine the properties of NGC 253. However, this reference distance has recently been convincingly revised to $3.5 \mathrm{Mpc}$ (Dalcanton et al. 2009). The final numerical values used below will therefore be needed to be scaled for consistency with the revised distance value.

Compared to the Milky Way, NGC 253 exhibits an increased overall star formation rate (SFR), with the SFR in the starburst nucleus being comparable to that in the entire remaining disk of the galaxy. An SN rate $v_{\mathrm{SN}}$ of this nucleus can be determined from the far-infrared (FIR) observations, since the FIR luminosity can be assumed to be directly proportional to $v_{\text {SN }}$ (Van Buren \& Greenhouse 1994). For NGC 253 as a whole the $\mathrm{SN}$ rate is estimated to be $\approx 0.08 \mathrm{yr}^{-1}$, with $\approx 0.03 \mathrm{yr}^{-1}$ in the starburst region (Engelbracht et al. 1998). By assessing the SFR, Melo et al. (2002) found that it can amount to $5 M_{\odot} \mathrm{yr}^{-1}$ in the starburst nucleus alone, which is $70 \%$ of the SFR of the entire galaxy. The starburst region itself has a cylindrical shape with a radius of $\approx 150 \mathrm{pc}$ and a full height of $\approx 60 \mathrm{pc}$ perpendicular to the disk of the galaxy and symmetric to its mid-plane with a volume $V_{\mathrm{SB}} \approx 1.2 \times 10^{62}(d / 2.6 \mathrm{Mpc})^{3} \mathrm{~cm}^{3}$ (Weaver et al. 2002).

To understand the observed $\gamma$-ray emission, a simplified scenario is considered in which the $\gamma$-ray production resulting from particle acceleration in the part of the disk outside the starburst region is neglected in comparison with that from the starburst region. The reasons are the low average gas density and radiation field intensity of the average interstellar medium (ISM) and the expected dominance of energy-dependent diffusive particle losses from the disk-quantitatively similar to the situation in the Milky Way. This expectation is also consistent with the estimate of Strong et al. (2010), who find that the HE $\gamma$ ray luminosity of the Milky Way is an order of magnitude lower than the $\gamma$-ray luminosity of the starburst region of NGC 253 (see Section 4).

The stellar winds from the early-type stars and the subsequent core-collapse SN explosions heat the lower-density parts of the surrounding material, causing them to expand rapidly from the starburst region in the form of a collective wind. The shocks from the SN explosions are the primary accelerators of CRs in 
this scenario, and their pressure adds to the excess thermal gas pressure. The dense material in the starburst region outside the SN remnants will remain essentially non-ionized in this process and will not participate in the flow. The $\pi^{0}$-producing CRs from the percolating wind flow are nevertheless likely to penetrate also the dense gas in the starburst region, which therefore is a massive target for $\gamma$-ray production. The recent detections of HE (Abdo et al. 2010a) and VHE $\gamma$-ray emission from the starburst galaxies NGC 253 (Acero et al. 2009) and M82 (Acciari et al. 2009) appear to support this picture.

In a picture where there is quasi-steady equilibrium between production and loss processes, the population of HE CRs accelerated in NGC 253's starburst nucleus is removed from the starburst region predominantly via three different processes: (1) advective removal of particles in the starburst wind, often also called a "superwind" (see, e.g., Weaver et al. 2002; Zirakashvili \& Völk 2006), not to be confused with the largescale galactic "disk wind" (see, e.g., Breitschwerdt et al. 1991; Heesen et al. 2009) that is primarily driven by the general population of CRs and the hot gas, both produced in the galactic disk; (2) diffusion of particles from the source region; and (3) catastrophic inelastic ("p-p") interactions. Energetic electrons/positrons suffer, in addition, radiative losses. The contributions of these components and the resulting $\gamma$-ray spectra have been discussed by several groups (see, e.g., Paglione et al. 1996; Aharonian et al. 2005; Domingo-Santamaría \& Torres 2005; Rephaeli et al. 2010; Lacki et al. 2010, 2011) and are compared to the measurements presented here.

After the discovery of VHE $\gamma$-ray emission from NGC 253 (Acero et al. 2009), we present for the first time a spectral analysis of the VHE $\gamma$-ray data obtained by H.E.S.S. in conjunction with the analysis of a 30 month set of Fermi-LAT data, increased in size by a factor of $\approx 3$ compared to the one used in the original publication of the Fermi Collaboration on NGC 253 (Abdo et al. 2010a). These results are used to estimate the properties of the underlying $\mathrm{CR}$ population, such as the particle energy density, as well as to place constraints on CR transport and the rms magnetic field strength within the starburst region.

\section{H.E.S.S. OBSERVATIONS AND DATA ANALYSIS}

\subsection{H.E.S.S. Instrument}

The High Energy Stereoscopic System (H.E.S.S.) is an array of four imaging atmospheric Cerenkov telescopes located in the Khomas Highland of Namibia, $1800 \mathrm{~m}$ above sea level. The telescopes are identical in construction, and each one comprises a $107 \mathrm{~m}^{2}$ optical reflector composed of segmented spherical mirrors and a camera built of 960 photomultiplier tubes. H.E.S.S. utilizes the imaging atmospheric Cerenkov technique (see, e.g., Hillas 1985). Cerenkov light, emitted by the highly relativistic charged particles in extensive air showers, is imaged by the mirrors onto the camera. A single shower can be recorded by multiple telescopes under different viewing angles, allowing stereoscopic reconstruction of the primary particle direction and energy with an average energy resolution of $15 \%$ and an event-by-event spatial resolution of 0.1 (Aharonian et al. 2006).

\subsection{Data Set}

NGC 253 was observed with the H.E.S.S. array in 2005 and from 2007 to 2009 for a total of $241 \mathrm{hr}$. After standard data
Table 1

VHE $\gamma$-ray Statistics of NGC 253

\begin{tabular}{llrrrrc}
\hline \hline Cuts & $\begin{array}{c}\theta_{\max }^{2} \\
\left(\mathrm{deg}^{2}\right)\end{array}$ & $N_{\text {On }}$ & $N_{\text {Off }}$ & $1 / \alpha$ & Excess & $\begin{array}{r}\text { Significance } \\
(\sigma)\end{array}$ \\
\hline$M A$ (standard) & 0.01 & 2240 & 26224 & 13.72 & $329 \pm 49$ & 7.1 \\
$M A$ (faint) & 0.005 & 571 & 7816 & 20.13 & $183 \pm 24$ & 8.4 \\
\hline
\end{tabular}

Notes. Number of $\gamma$-ray-like and background events along with the significance (Li \& Ma 1983) and $\gamma$-ray excess as obtained for the H.E.S.S. data using the $M A$ method. $\alpha$ denotes the normalization factor between signal and background exposures.

quality selection, where data taken under unstable weather conditions or with malfunctioning hardware have been excluded, the total live time amounts to $177 \mathrm{hr}$ of three- and four-telescope observations that were used for the generation of sky maps of the $\gamma$-ray emission and the reconstruction of energy spectra. Observations were carried out at zenith angles of $1^{\circ}-42^{\circ}$, with a mean value of $12^{\circ}$. Observations have been performed in the wobble mode, where the telescopes were alternately pointed offset in R.A. and decl. from NGC 253, resulting in an average pointing offset of 0.5 (Aharonian et al. 2006).

\subsection{Data Analysis}

All results presented in the following were obtained using the model analysis (MA; de Naurois \& Rolland 2009) for event reconstruction and background reduction and were crosschecked with the boosted-decision-tree-based (BDT) Hillas parameter technique described in detail in Ohm et al. (2009). Two different sets of cuts were used for $M A$ : the standard cuts require a minimum shower image intensity of 60 p.e. in each camera. They maximize the acceptance of $\gamma$-ray-like events (at the expense of a larger background) and are therefore used for energy spectra and sky maps; the faint cuts, requiring a higher minimum intensity of 120 p.e., resulting in improved angular resolution at the cost of lower $\gamma$-ray acceptance, are used for position and extension determination. Spectral results were derived using the Reflected background model, whereas the Ring background model was utilized to generate sky maps (Berge et al. 2007). The analysis thresholds for the standard and faint cuts configuration are $190 \mathrm{GeV}$ and $250 \mathrm{GeV}$ for the $M A$ method, respectively.

\subsection{Results}

A VHE $\gamma$-ray excess map for the $1.5 \times 1.5$ field of view (FoV) centered on the optical position of NGC 253 and produced with $M A$, standard cuts, is shown in Figure 1. The map has been smoothed with a two-dimensional Gaussian kernel of 3.9 rms to reduce the effect of statistical fluctuations and matched to the point-spread function (PSF) for this analysis. A total of $329 \pm 49$ excess events corresponding to a significance of $7.1 \sigma$ (Li \& Ma 1983) are found at the nominal position of NGC 253. The overall statistics of $M A$ with both sets of cuts are shown in Table 1. The best-fit position of the source is R.A. $00^{\mathrm{h}} 47^{\mathrm{m}} 34^{\mathrm{s}} .3 \pm 1^{\mathrm{s}} \mathrm{s}$, decl. $-25^{\circ} 17^{\prime} 22^{\prime \prime} .6 \pm 0$. 3 (J2000), compatible at the $<1 \sigma$ level with the optical center of NGC 253 at R.A. $00^{\mathrm{h}} 47^{\mathrm{m}} 33^{\mathrm{s}} .1$ and decl. $-25^{\circ} 17^{\prime} 18^{\prime \prime}$ (J2000).

The squared angular distribution of $\gamma$-ray candidate and background events relative to the position of NGC 253 as shown in Figure 2 is consistent with point-like emission. This constrains a potential source extension to less than 2.4 at $3 \sigma$ confidence level (e.g., the extent of the starburst region in 
Table 2

H.E.S.S. Spectral Results of NGC 253

\begin{tabular}{lccc}
\hline \hline Data & $\begin{array}{c}E_{\mathrm{th}} \\
(\mathrm{GeV})\end{array}$ & $\begin{array}{c}F_{0} \\
\left(\mathrm{TeV}^{-1} \mathrm{~cm}^{-2} \mathrm{~s}^{-1}\right)\end{array}$ & $\begin{array}{c}F\left(>E_{\mathrm{th}}\right) \\
\left(\mathrm{cm}^{-2} \mathrm{~s}^{-1}\right)\end{array}$ \\
\hline This analysis by $M A$ & 190 & $(9.6 \pm 1.5) \times 10^{-14}$ & $(5.6 \pm 1.2) \times 10^{-13}$ \\
Prev. analysis (Acero et al. 2009) & 220 & & $2.14 \pm 0.18$ \\
\hline
\end{tabular}

Notes. VHE $\gamma$-ray spectral results as shown in Figure 3 for the $M A$. The photon index $\Gamma$ is derived from a fit of a power law to the spectrum. Only statistical errors are given. A comparison with the integral flux given in Acero et al. (2009) is also provided.

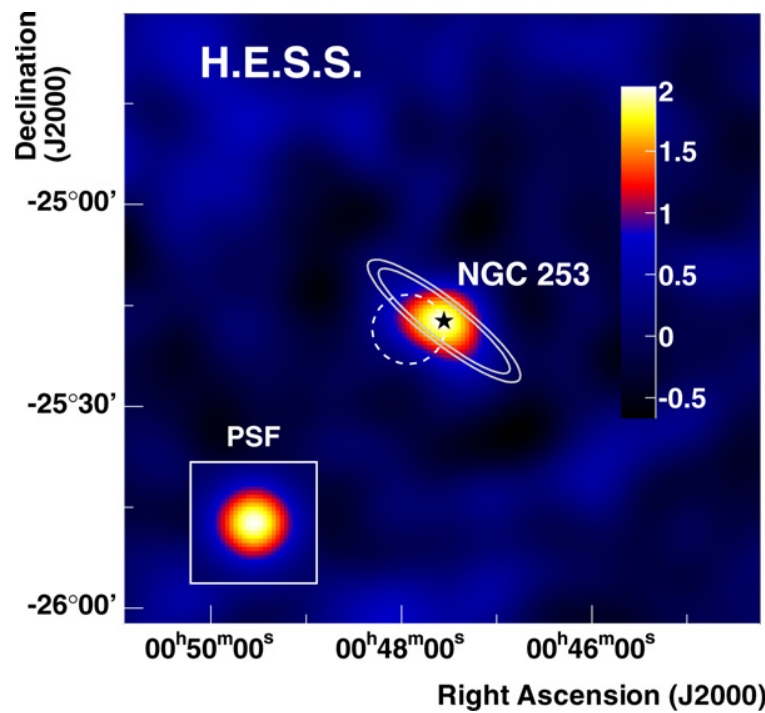

Figure 1. Smoothed H.E.S.S. $\gamma$-ray excess map in units of VHE $\gamma$-ray events per $\operatorname{arcmin}^{2}$ of the $1.5 \times 1.5 \mathrm{FOV}$, centered on the position of NGC 253. The image was smoothed with a Gaussian kernel of 3.9 rms, the radius that corresponds to the PSF for this analysis. The black star marks the position of the optical center of NGC 253, and the inlay represents the size of a point-like source as it would have been seen by H.E.S.S. for this analysis. White contours depict the optical emission from the whole galaxy with contour levels of constant surface brightness of $25 \mathrm{mag} \operatorname{arcsec}^{-2}$ and $23.94 \mathrm{mag} \mathrm{arcsec}^{-2}$ as used in Pence (1980). The dashed circle indicates the $95 \%$ error contour of the best-fit position of the Fermi-LAT source (see also Table 3).

(A color version of this figure is available in the online journal.)

${ }^{12} \mathrm{CO}(2-1)$ is about $\approx 0.4 \times 1{ }^{\prime} .0$; Sakamoto et al. 2011). In the standard picture, where the HE and VHE $\gamma$-ray emission from starburst galaxies originates from diffuse CR interactions, no variability of the $\gamma$-ray signal is expected. The yearly light curve of the $\gamma$-ray emission is stable over the four years of observations within errors. A fit of a constant flux to the yearly light curve yields a mean integral flux above $1 \mathrm{TeV}$ of $(8.5 \pm 1.8) \times 10^{-14} \mathrm{~cm}^{-2} \mathrm{~s}^{-1}$ with a $\chi^{2}$ of 3.3 for 3 degrees of freedom and is stable over the four years of observations within errors.

The H.E.S.S. data set previously published in Acero et al. (2009) comprises $119 \mathrm{hr}$ of good-quality data, collected in the years 2005, 2008, and 2009, and represents 2/3 of the data set used in this work. Based on this larger data set, a better determination of the spectral characteristics is now possible. All results presented in that publication are consistent with the findings presented here.

\subsection{Spectrum}

The differential energy spectrum derived from this data set is shown in Figure 3 and Table 2 and is well described by a power law: $d N / d E=F_{0} \cdot(E / 1 \mathrm{TeV})^{-\Gamma}$ with photon

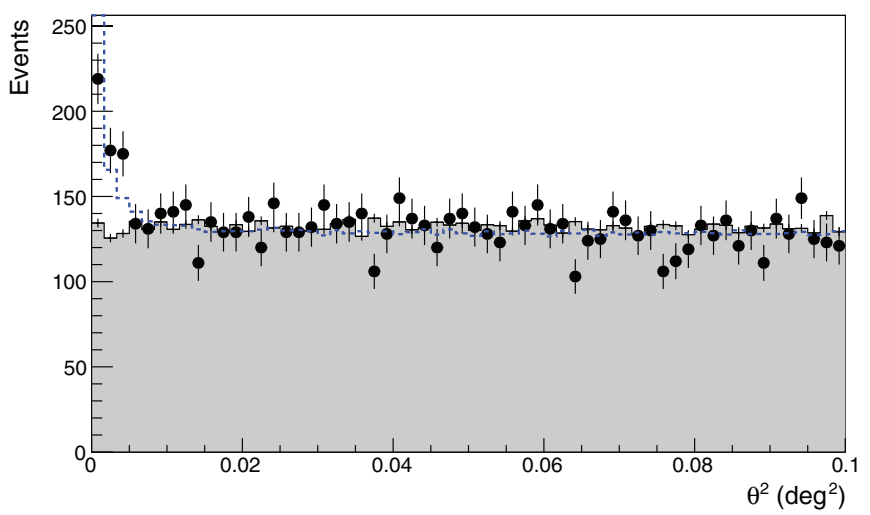

Figure 2. Distribution of ON events around NGC 253 and of OFF events from background control regions as obtained with $M A$, faint source cuts. The squared angular distribution has been produced using the Reflected background model. Also shown is the point-spread function of the instrument for this analysis (dotted line), assuming that the $\gamma$-ray emission originates from the optical center of NGC 253. The normalization of the model is adjusted to match the total $\gamma$-ray excess in the range $0-0.01 \mathrm{deg}^{2}$.

(A color version of this figure is available in the online journal.)

index $\Gamma=2.14 \pm 0.18_{\text {stat }} \pm 0.30_{\text {sys }}$ and flux normalization $F_{0}=\left(9.6 \pm 1.5_{\text {stat }}(+5.7,-2.9)_{\text {sys }}\right) \times 10^{-14} \mathrm{TeV}^{-1} \mathrm{~cm}^{-2} \mathrm{~s}^{-1}$ with a chance probability of $7 \%$. This yields an integral flux above the energy threshold of $190 \mathrm{GeV}$ of $F(>190 \mathrm{GeV})=$ $(5.6 \pm 1.2) \times 10^{-13} \mathrm{~cm}^{-2} \mathrm{~s}^{-1}$. With a flux of $(0.21 \pm 0.05) \%$ of the Crab Nebula flux above the energy threshold, NGC 253 is the source with the lowest VHE $\gamma$-ray flux detected so far.

Since the VHE $\gamma$-ray signal from NGC 253 is so weak, systematic effects could potentially influence the spectral reconstruction. In-depth systematic checks on the background subtraction and the spectrum calculation have been performed with the $M A$ and BDT method. These tests have been used to estimate the systematic uncertainty of the VHE $\gamma$-ray spectrum presented here. For instance, the difference in the data selection procedures leads to a difference in live time of $12 \%$ and hence to a small difference in the data sets used to reconstruct the spectrum. Furthermore, Earth's magnetic field bends charged particles in extensive air showers and influences their development. This affects observables such as the shower image orientation in the Cerenkov camera, the image shape, and hence the stereoscopic reconstruction. Although this effect is expected to be small, it is taken into account in the reconstruction of the energy spectrum of NGC 253. In order to test the background systematics, harder selection cuts on $\gamma$-ray-like events have been performed, e.g., the faint cuts introduced in Section 2.3, and alternate background estimation techniques (such as the template background) have been studied as well. Precise comparison between the actual level of background in the whole field of view (FOV) and predictions from the background model, excluding a circle of 0.25 around NGC 253, indicates that the background level is 


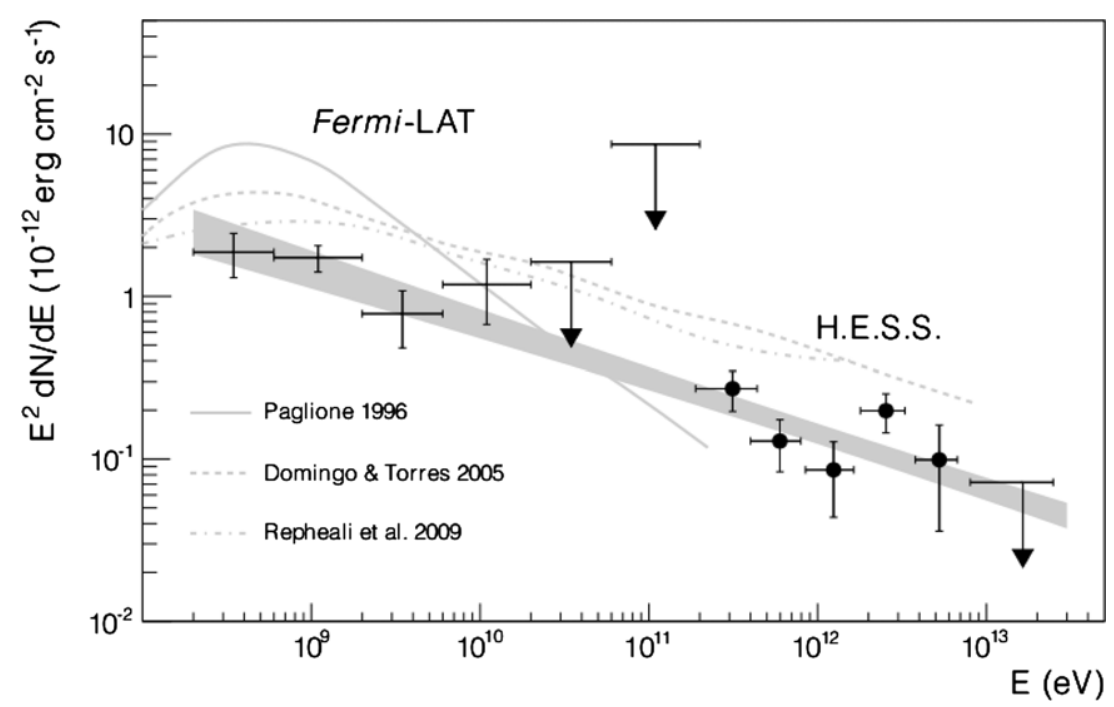

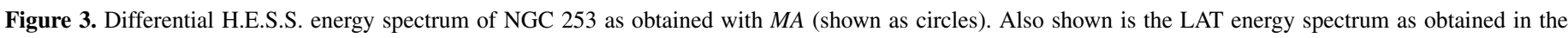

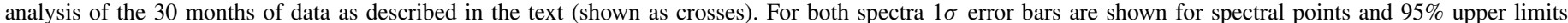

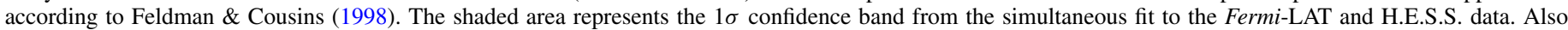
shown are the predictions from Paglione et al. (1995; solid gray), Domingo-Santamaría \& Torres (2005; dashed gray), and Rephaeli et al. (2010; dash-dotted gray).

controlled at a level of $\approx 1.5 \%$ over the full FOV, with the magnetic field introducing an azimutal asymmetry of $\pm 2 \%$. The magnetic field and potential systematic effects in the background determination mainly affect low-energy events. Therefore, additional tests have been performed, where only events with reconstructed energies above $0.6 \mathrm{TeV}$ have been used in the spectral fit. All systematic tests have been performed with both analysis chains and resulted in a spread in normalization between the two analyses of $+60 \%,-30 \%$ and a variation in differential photon index of $\Delta \Gamma_{\text {sys }} \leqslant 0.3$.

\section{FERMI-LAT OBSERVATIONS AND DATA ANALYSIS}

Based on 11 months of data, NGC 253 has also been detected in the HE $\gamma$-ray regime by the Fermi-LAT instrument (Abdo et al. 2010a). It was later confirmed as an HE $\gamma$-ray emitter based on 12 and 24 months of data (Abdo et al. 2010b; Nolan et al. 2012). The analysis and the results of a larger 30 month data set are presented in the following.

\subsection{Fermi-LAT instrument}

The Fermi-LAT instrument is a pair-conversion telescope, capable of detecting $\gamma$-rays in the energy range between $20 \mathrm{MeV}$ and $300 \mathrm{GeV}$. It consists of a tracker for the reconstruction of the particle direction, a calorimeter that measures the energy of the incident particle, and an anti-coincidence system designed to suppress the charged-particle background. Data are normally recorded in survey mode, in which the whole sky is covered every two orbits. The instrument FOV is $\approx 2.4 \mathrm{sr}$, and it provides an angular resolution of $<1^{\circ}$ at $1 \mathrm{GeV}$ and $<0.2$ at $10 \mathrm{GeV}$. A full description of the mission- and instrument-related details can be found in Atwood et al. (2009).

\subsection{Data Set and Data Analysis}

The data set presented in the following comprises data from the commissioning of Fermi on 2008 August 4 (MJD 54,682) until 2011 February 3 (MJD 55,595). The data analysis has been performed using events with reconstructed energies between $200 \mathrm{MeV}$ and $200 \mathrm{GeV}$ and utilizing the Fermi Science Tools
(FST) package, version v9r18p6. ${ }^{35}$ Events of the "diffuse" source class have been analyzed using the P6_V3_Diffuse instrument response functions (IRFs). Additionally, events with zenith angles $>105^{\circ}$ were excluded from the analysis due to a significant contribution of Earth-limb $\gamma$-rays. ${ }^{36}$

The best-fit position of NGC 253 has been determined by means of a maximum likelihood method, using the FST tool gtfindsrc. All events in a region of interest (ROI) of $10^{\circ}$ around this best-fit position have been used, and all sources within $15^{\circ}$ were modeled to produce energy spectra and to calculate the test statistic $(\mathrm{TS})^{37}$ of the source. At the best-fit position at R.A. $00^{\mathrm{h}} 47^{\mathrm{m}} 55^{\mathrm{s}} .7$ and decl. $-25^{\circ} 18^{\prime} 32^{\prime \prime} .4(\mathrm{~J} 2000)\left(r_{95}=5.7\right.$, $95 \%$ confidence level) a TS value of 105 , corresponding to a statistical significance of $\approx 10 \sigma$, is found.

Using the FST gtlike tool and an unbinned maximum likelihood fitting procedure, the energy spectrum of NGC 253 has been derived. For this purpose, all sources listed in the FermiLAT 1 year catalog in the $15^{\circ}$ region around the best-fit position were modeled with a power law $\left(d N / d E \propto E^{-\Gamma}\right)$, with differential photon index $\Gamma$. In a second step, residual sources in the TS map in the ROI with a TS $>16$ were included in the model as well. Compared to the 1 year and 2 year catalog, 20 and 16 more candidates, respectively, are included in the source model used in this work. In the minimization procedure, the integral flux in the energy range of interest and the photon index were left as free parameters for all sources in the ROI and fixed for all sources between $10^{\circ}$ and $15^{\circ}$. The diffuse Galactic and extragalactic background components were modeled with the files gll_iem_v02.fit and isotropic_iem_v02.txt, ${ }^{38}$ respectively. The energy spectrum is best described by a power law in energy with $\Gamma=2.24 \pm 0.14_{\text {stat }} \pm 0.03_{\text {sys }}$ and an integral flux of $F(0.2-200 \mathrm{GeV})=\left(4.9 \pm 1.0_{\text {stat }} \pm 0.3_{\text {sys }}\right) \times 10^{-9} \mathrm{~cm}^{-2} \mathrm{~s}^{-1}$.

\footnotetext{
35 http://fermi.gsfc.nasa.gov/ssc/data/analysis/documentation/Cicerone/

36 Note that as a cross-check, the data have also been analyzed with the more recent FST release, version v9r23p1, and the P7Source_V6 IRFs and found to give consistent results.

$37 \sqrt{\mathrm{TS}}$ is a measure of the significance and is defined as

$\mathrm{TS}=-2 \Delta \log$ (likelihood) between models including and excluding the source (Mattox et al. 1996).

38 http://fermi.gsfc.nasa.gov/ssc/data/access/lat/BackgroundModels.html
} 
Table 3

Fermi-LAT Spectrum and Position of NGC 253

\begin{tabular}{lccccc}
\hline \hline $\begin{array}{l}\text { R.A. (J2000) } \\
(\mathrm{deg})\end{array}$ & $\begin{array}{c}\text { Decl. (J2000) } \\
(\mathrm{deg})\end{array}$ & $\begin{array}{c}r_{95} \\
(\mathrm{deg})\end{array}$ & $\begin{array}{c}F(0.2-200 \mathrm{GeV}) \\
\left(10^{-9} \mathrm{~cm}^{-2} \mathrm{~s}^{-1}\right)\end{array}$ & $\Gamma$ & TS \\
\hline 11.982 & -25.309 & 0.095 & $4.9 \pm 1.0_{\text {stat }} \pm 0.3_{\text {sys }}$ & $2.24 \pm 0.14_{\text {stat }} \pm 0.03_{\text {sys }}$ & 105 \\
\hline
\end{tabular}

Notes. Best-fit position and results of the maximum likelihood analysis between $200 \mathrm{MeV}$ and $200 \mathrm{GeV}$.

Table 4

Fermi-LAT Spectral Results of NGC 253

\begin{tabular}{lcc}
\hline \hline $\begin{array}{l}E_{\min }-E_{\max } \\
(\mathrm{GeV})\end{array}$ & $\begin{array}{c}F_{\mathrm{NGC} \mathrm{253}} \\
\left(10^{-12} \mathrm{erg} \mathrm{cm}^{-2} \mathrm{~s}^{-1}\right)\end{array}$ & $\begin{array}{c}\text { Syst. Uncer. } \\
(\%)\end{array}$ \\
\hline $0.2-0.6$ & $1.9 \pm 0.6$ & 10 \\
$0.6-2.0$ & $1.7 \pm 0.3$ & 10 \\
$2.0-6.0$ & $0.8 \pm 0.3$ & 15 \\
$6.0-20.0$ & $1.2 \pm 0.5$ & 20 \\
$20.0-60.0$ & $<1.7$ & 20 \\
$60.0-200.0$ & $<8.7$ & 20 \\
\hline
\end{tabular}

Notes. HE $\gamma$-ray spectral results of the maximum likelihood analysis in energy bands as shown in Figure 3. $1 \sigma$ statistical errors and 95\% upper limits are given. Systematic uncertainties have been obtained using the bracketing method (see, e.g., Abdo et al. 2009).

The positional and spectral results of the likelihood analysis are summarized in Table 3.

The flux in each energy band as summarized in Table 4 and shown in Figure 3 has been reconstructed in the same way as for the full energy range, where the photon index and integral flux in that band were left as free parameters again. Note that all spectral points agree within $1 \sigma$ statistical error with the spectral points and upper limits as reported in Abdo et al. (2010a).

The systematic uncertainty on the total spectrum has been estimated following the bracketing method (e.g., Abdo et al. 2009), where the effective area is shifted up- and downward according to its systematic uncertainty in the corresponding energy range. Following Abdo et al. (2010b), the systematic uncertainty on the flux as inferred by the systematic uncertainty of the effective area is of the order of $10 \%$ at $100 \mathrm{MeV}, 5 \%$ at $0.5 \mathrm{GeV}$, and $20 \%$ at $10 \mathrm{GeV}$. As for the VHE $\gamma$-ray light curve, there is no significant variability seen in the yearly $\mathrm{HE} \gamma$-ray emission

\section{THE HE-VHE $\gamma$-RAY SPECTRUM}

Within the $1 \sigma$ statistical errors, the H.E.S.S. measurement and the Fermi results are compatible, in respective value of photon index as well as respective normalization. A simultaneous, single power-law fit to the H.E.S.S. and Fermi-LAT spectral points results in a photon index of $\Gamma_{\text {sim }}=2.34 \pm 0.03$ and an energy flux at $1 \mathrm{GeV}$ of $(1.5 \pm 0.2) \times 10^{-12} \mathrm{erg} \mathrm{cm}^{-2} \mathrm{~s}^{-1} .39$ This corresponds to an integral energy flux above $0.2 \mathrm{GeV}$ of $F_{\chi \text { meas }}(>0.2 \mathrm{GeV}) \simeq 5.3 \times 10^{-12} \mathrm{erg} \mathrm{cm}^{-2} \mathrm{~s}^{-1}$. The fit has a $\chi^{2}$ of 8.27 for 7 degrees of freedom and a $p$-value of $30 \%$. Note that the somewhat steeper index for the simultaneous fit compared to the individual measurements is fully compatible within the $1 \sigma$ statistical uncertainties of the Fermi and H.E.S.S. photon index. Even when taking into account a 30\% downward shift to account for the systematic error in reconstructed normalization

\footnotetext{
39 Note that no forward-folding technique has been applied and the fit is performed only taking into account statistical errors.
}

of the VHE $\gamma$-ray spectrum as presented in Section 2.5, the single power-law fit has a $p$-value of $8.8 \%$.

The fit of a broken power law to the HE and VHE $\gamma$ ray points with a break energy between 10 and $300 \mathrm{GeV}$ (between H.E.S.S. and Fermi) results in a best-fit index change of $\Delta \Gamma=0.1 \pm 0.3$ with break energy at $300 \mathrm{GeV}$. Although a photon index change between the Fermi and H.E.S.S. energy range cannot be excluded, the hypothesis of no index change is favored given the available data. This result suggests that in order to explain the available data no spectral model that includes a break or turnover is needed.

The integral (HE-VHE) flux above $200 \mathrm{MeV}$ corresponds to a $\gamma$-ray luminosity of $L_{\gamma}^{E}(>200 \mathrm{MeV}) \simeq 7.8 \times 10^{39} \mathrm{erg} \mathrm{s}^{-1}$, for a distance of $3.5 \mathrm{Mpc}$. This is about a factor of 10 larger than the $\gamma$-ray luminosity $L_{\gamma}^{\mathrm{MW}}(>200 \mathrm{MeV}) \simeq(7-10) \times 10^{38} \mathrm{erg} \mathrm{s}^{-1}$ for the Milky Way, as estimated by Strong et al. (2010).

\section{DISCUSSION}

The results presented in the previous sections have a number of interesting implications for the nature and properties of the $\gamma$-ray source in the starburst nucleus of NGC 253. Note that the discussion is based on the observationally favored result that the spectrum of NGC 253 can be described by a single power law from the HE to the VHE $\gamma$-ray regime. Quantities used in the following are summarized in Table 5. This section is organized as follows: First, the aspect of dominance of a hadronic scenario is discussed. Second, arguments for an energy-independent particle transport in the starburst region are given, the consequences of this picture are explored, and limits on the diffusion coefficient are presented. Third, the $\gamma$-ray flux estimated from the $\mathrm{SN}$ rate is compared to the experimental results. Fourth, the magnetic field in the starburst region is estimated. And finally, the contribution of discrete sources to the $\gamma$-ray luminosity of NGC 253 is assessed.

\subsection{Dominance of Hadronic $\gamma$-ray Emission}

Early model predictions for the various contributions to the overall HE and VHE $\gamma$-ray spectral energy distribution (e.g., Paglione et al. 1996) and subsequent more detailed calculations (e.g., Domingo-Santamaría \& Torres 2005; Rephaeli et al. 2010; Lacki et al. 2010, 2011) all consider diffuse $\gamma$-ray emission and neglect the contributions of discrete sources. They are roughly compatible with the observational results. Three such model curves are shown in Figure 3.

These largely phenomenological models use primary electron-to-proton ratios similar to those inferred for the ISM of the Milky Way and parameterize the diffusive escape times from the starburst region, while assuming advection speeds of the order of several hundred $\mathrm{km} \mathrm{s}^{-1}$. Also the secondary leptonic component from the decay of charged pions is included in the calculation of the expected bremsstrahlung and inverse Compton $\gamma$-ray emission. In detail, the three model spectra in Figure 3 are based on very different assumptions regarding 
Table 5

Symbols, Units, and Descriptions of Quantities Used in the Discussion

\begin{tabular}{lcl}
\hline \hline Symbol & Units & \multicolumn{1}{c}{ Description } \\
\hline$\epsilon$ & $\mathrm{GeV}$ & Energy of individual particle \\
$E_{\mathrm{pp}}^{\pi}$ & $\mathrm{GeV}$ & Total non-thermal energy of pion-producing particles \\
$\tilde{Q}$ & $\mathrm{GeV}^{-1} \mathrm{~cm}^{-3} \mathrm{~s}^{-1}$ & Differential volumetric particle source term \\
$Q$ & $\mathrm{GeV} \mathrm{s}^{-1}$ & Volume-integrated particle source term \\
$Q^{\pi}$ & $\mathrm{GeV} \mathrm{s}^{-1}$ & Volume-integrated source term of pion-producing particles
\end{tabular}

the relative magnitudes of the advective $\left(\tau_{\mathrm{ad}}\right)$ and energydependent diffusive $\left(\tau_{\text {diff }}\right)$ particle escape times on the one hand and on the assumed source spectra on the other. These models assume $\tau_{\text {diff }}=\tau_{0}\left(\epsilon_{\mathrm{p}} /(1 \mathrm{GeV})^{-0.5}\right.$, where $\epsilon_{\mathrm{p}}$ is the proton energy. In Paglione et al. (1996), $\tau_{\mathrm{ad}}>\tau_{\text {diff }}$ for all energies observed. ${ }^{40}$ With an assumed proton differential source spectral index $s=2.2$, the $\gamma$-ray spectral energy density (SED) has a rather steep dependence $\propto \epsilon_{\gamma}^{-0.7}$ on the $\gamma$-ray energy $\epsilon_{\gamma}$ due to the dominance of diffusive escape. Rephaeli et al. (2010) obtain $\tau_{\mathrm{ad}}>\tau_{\text {diff }}$. However, their source spectrum is assumed to be quite hard $(s=2.0)$. These authors model the emission from the galactic disk as a whole and find approximately an SED $\propto \epsilon_{\gamma}^{-0.35}$ (see their Figure 3). No spatial profiles are given. Domingo-Santamaría \& Torres (2005) use for their main plot $\tau_{0}=10 \mathrm{Myr}$ and $\tau_{\mathrm{ad}}=0.3 \mathrm{Myr}$, resulting in $\tau_{\mathrm{ad}}<\tau_{\text {diff }}$ for $\epsilon_{\mathrm{p}} \leqslant 1 \mathrm{TeV}$. Therefore, their SEDs for low $\gamma$-ray energies should be advection-dominated and correspond to the source spectrum. The corresponding curves in their Figures 5 and 9 indicate this source spectrum for an assumed particle source index $s \simeq 2.3$ up to $\epsilon_{\gamma}$ equal to some hundreds of $\mathrm{GeV}$. These curves indicate a significant softening beyond about $1 \mathrm{TeV}$. For a smaller value of $\tau_{0}$ their SED should fall off strongly $\propto \epsilon_{\gamma}^{-(s-2)-0.5}$ already beyond some considerably lower value of $\epsilon_{\gamma}$.

The above model results show hadronically dominated $\gamma$-ray emission up to several $\mathrm{TeV}$, where the $\gamma$-rays are primarily produced in inelastic collisions between nuclear CRs and target nuclei from the ambient ISM and subsequent $\pi^{0}$ decay. Bremsstrahlung and inverse Compton emission from primary and secondary electrons is not entirely negligible and in some of these models only by a factor of a few below the hadronic emission. This is in particular the case for $\gamma$-ray energies below $100 \mathrm{MeV}$, where the $\pi^{0}$-decay emission drops off, and at the $\mathrm{HE}$ end, where the harder inverse Compton emission in the Thomson limit can win over the $\pi^{0}$-decay emission (if electrons at these energies do not experience significant radiative losses). Note, however, that the ratio of hadronic to leptonic emission depends on the assumed source spectrum, the form of $\tau_{\text {diff }}\left(\epsilon_{\mathrm{p}}\right)$, and the assumed electron-to-proton ratio (which has to be $\gg$ than the canonical value of $1 / 100$ that electrons dominate over the hadronic component and overcome energetics problems; see, e.g., Ohm \& Hinton 2012). Therefore, it is very likely that the hadronic emission dominates over the leptonic emission. That this is possible is basically a consequence of the very high gas density $n_{\mathrm{g}}$ in the starburst region. Aharonian et al. (2005) find an $n_{\mathrm{g}}=580 \mathrm{~cm}^{-3}$ for a total gas mass of $6 \times 10^{7} \mathrm{M}_{\odot}$, the value preferred by Engelbracht et al. (1998). Lower and higher gas masses as found by Mauersberger et al. (1996) and Sorai et al. (2000), respectively, imply an uncertainty of this estimate of $\approx 20 \%$. Note that this estimate only takes into

\footnotetext{
40 Due to a misprint in that paper, the $\tau_{0}$ in their Figure 6 is in fact $1 \mathrm{Myr}$ instead of the quoted $\tau_{0}=10 \mathrm{Myr}$ (T. A. D. Paglione 2012, private communication).
}

account the uncertainty in total gas mass and neglects any uncertainty in the starburst volume due to possible projection effects. Clearly this density is an average value-the actual gas density is probably extremely inhomogeneous, given the large localized energy inputs from stars and in particular from their $\mathrm{SN}$ explosions. Therefore, the local density for $\mathrm{p}-\mathrm{p}$ interactions could differ from the average density. However, it is unlikely that protons escape from the denser regions without significant losses. Adopting this $n_{\mathrm{g}}$ corresponds to an average loss time due to inelastic $\mathrm{p}-\mathrm{p}$ collisions of $\bar{\tau}_{\mathrm{pp}} \approx 1.1 \times 10^{5} \mathrm{yr}$ (this value is mildly dependent on the observed spectral index due to the energy dependence of the $\mathrm{p}-\mathrm{p}$ cross-section $\sigma_{\mathrm{pp}}$; a derivation of this value is given in the Appendix). Finally, it has to be noted that $\bar{\tau}_{\mathrm{pp}}$ scales inversely linear with the inelasticity factor of the $\mathrm{p}-\mathrm{p}$ collision (see the Appendix). This can introduce an additional uncertainty on $\bar{\tau}_{\mathrm{pp}}$ of $\approx 20 \%$.

We conclude that the $\gamma$-ray emission from the starburst region is likely to be dominated by hadronic interactions. In the following we will discuss the impact of system parameters such as $\mathrm{SN}$ rate, outflow velocity, and particle diffusion coefficient on the $\gamma$-ray emission within the hadronic scenario. The possible additional role of discrete $\gamma$-ray sources will be briefly discussed in Section 5.6.

\subsection{Cosmic-ray Escape}

In the hadronically dominated case, there are three main scenarios for the expected $\gamma$-ray emission, depending on the (potentially energy dependent) probability of CRs escaping the starburst region. In the case that the escape probability is $\ll 1$ at all energies, the system can be said to be calorimetric. The measured $\gamma$-ray flux is significantly lower than the flux expected in the calorimetric limit for the canonical parameters, such as the CR acceleration efficiency as used in Section 5.4. CR escape must therefore be considered. There are two competing mechanisms to remove CRs from the system: diffusion and advection. Dominance of advection (which is an energy-independent process) over the full energy range would result in a $\gamma$-ray spectrum that approximately resembles the source spectrum. Diffusion, on the other hand, is an energy-dependent process and would lead to a spectral steepening of the source spectrum. At very low energies, the diffusion loss timescale $\tau_{\text {diff }}$ becomes very long and eventually comparable to the advective loss timescale $\tau_{\mathrm{ad}}$. At the critical energy, where the diffusive transport takes over from advective transport and $\tau_{\text {diff }}=\tau_{\text {ad }}$, a spectral break in the source spectrum is expected.

Empirically, for the Milky Way, the measurements of the CR secondary-to-primary ratio (Strong et al. 2007) suggest a softening of the CR source spectrum by a factor $\propto E^{-\alpha}$, with $0.3 \lesssim \alpha \lesssim 0.6$, above a few $\mathrm{GeV}$ per nucleon as a result of energy-dependent diffusion. This is consistent with a comparatively modest production rate of hot gas and CRs per unit area of the Galactic disk that allows most of the thermal gas to cool radiatively. At the same time, the excitation of magnetic 
field fluctuations by the escaping CR particles and their coupling to the thermal gas is equally modest. Although a CR-driven wind will develop at large distances from the disk, the observed particle spectrum in the Galactic disk is dominated by diffusive escape (Ptuskin et al. 1997). On the other hand, the wind from the starburst region in NGC 253 is very strong as a result of very strong gas heating that cannot be compensated by radiative cooling. The energy flux density in CRs driving magnetic fluctuations is expected to be very large as well, given the high SN rate in the small starburst volume (see, e.g., Aharonian et al. 2005, and Section 5.5). These nonlinear effects massively diminish the role of particle diffusion relative to global advection in the wind. Indeed, as shown in the next section, the observed $\gamma$-ray flux $F_{\gamma}^{\text {meas }}(>200 \mathrm{MeV})$ is well explained quantitatively by pure particle advection in the wind from the starburst nucleus, which requires a $C R$ production $\Theta E_{\mathrm{SN}} \simeq 10^{50} \mathrm{erg}$ per $\mathrm{SN}$ (e.g., Drury et al. 1989) for the starburst parameters of NGC 253.

The diffusion time in NGC 253's starburst nucleus can be expressed as $t_{\text {diff }}=(H / 2)^{2} \kappa^{-1}$, where $\kappa$ denotes the diffusion coefficient and $H \approx 60 \mathrm{pc}$ is the height of the starburst region, positioned symmetrically to the galactic mid-plane. If $t_{\mathrm{ad}} \lesssim t_{\text {diff }}$ is required, then $\kappa$ should not exceed about $3 \times 10^{27} \mathrm{~cm}^{2} \mathrm{~s}^{-1}$ for all energies below the last H.E.S.S. flux point at $4.7 \mathrm{TeV}$ that corresponds to a CR energy of $\approx 30 \mathrm{TeV}$ (see, e.g., Kelner et al. 2006). Such a small diffusion coefficient could be the result of strong wave excitation by the exceedingly concentrated CR production in the small starburst volume (Aharonian et al. 2005). This value can be compared to that of Bohm diffusion, which should be considered the slowest possible form of diffusion for a randomized magnetic field configuration. The Bohm limit is given by $\kappa_{\mathrm{Bohm}} \approx 3 \times 10^{22}\left(\epsilon_{\mathrm{GeV}} / B_{\mu \mathrm{G}}\right) \mathrm{cm}^{2} \mathrm{~s}^{-1}$, where $\epsilon_{\mathrm{GeV}}$ is the $\mathrm{CR}$ energy in $\mathrm{GeV}$ and $B_{\mu \mathrm{G}}$ is the magnetic field in $\mu \mathrm{G}$. For a particle energy of $30 \mathrm{TeV}$ and a magnetic field of $100 \mu \mathrm{G}$, as estimated in the next section, the Bohm diffusion coefficient is about $3 \times 10^{24} \mathrm{~cm}^{2} \mathrm{~s}^{-1}$ and thus still three orders of magnitude smaller than the maximum diffusion coefficient deduced from the single power-law spectrum of the $\gamma$-ray data. The upper limit to the diffusion coefficient estimated for the starburst region in NGC 253 can also be compared to the average value inferred for the Milky Way which is given by $\kappa_{\text {gal }} \approx 1.5 \times 10^{30}(E / 1 \mathrm{TeV})^{1.1} \mathrm{~cm}^{2} \mathrm{~s}^{-1}$ (Ptuskin et al. 1997; Aharonian et al. 2005). With such a comparison, extrapolating to a particle energy of $30 \mathrm{TeV}, \kappa \lesssim 5 \times 10^{-5} \kappa_{\text {gal }}$ is found. This is an interesting requirement on the scattering strength of the magnetic field fluctuations ${ }^{41}$ in the starburst region, but certainly not an outrageous one, comparing with the Bohm scattering level.

Dominant transport by advection is suggested by the observation of a rather hard $\gamma$-ray spectrum and the smooth alignment of the spectrum in the $\mathrm{HE}$ and the VHE $\gamma$-ray regime - the most noticeable observational result of this paper (see Section 4). Therefore, taking the combined Fermi and H.E.S.S. spectra as a single power law as indicated in Figure 3 with differential index $\Gamma_{\text {sim }}=2.34$ argues that energy-dependent diffusion is not important in the energy range covered. ${ }^{42}$ In fact, advection alone already explains the magnitude of $F_{\gamma}^{\text {meas }}$ quantitatively.

\footnotetext{
41 This value can also be compared to the gyroradius of a proton with $1 \mathrm{TeV}$ energy in a $100 \mu \mathrm{G}$ field, which is $r_{g} \approx 3.3 \times 10^{13}\left(\epsilon_{\mathrm{TeV}} / B_{100 \mu \mathrm{G}}\right) \mathrm{cm} \approx 2 \mathrm{AU}$. 42 Energy-independent diffusion can in principle occur as a result of large-scale turbulent motions in the gas and may also contribute to energetic-particle confinement in the gas that is systematically streaming with $v_{\text {wind }}$ (e.g., Bykov 2001; Parizot et al. 2004). This effect is not included here explicitly.
}

Therefore, two conclusions may be drawn here: (1) the observed spectrum is most likely the result of an energyindependent transport mechanism, i.e., advection and adiabatic expansion in the starburst wind and inelastic nuclear energy losses, and (2) the combined observed $\gamma$-ray spectrum in the $\mathrm{HE}$ and VHE $\gamma$-ray regimes might in this case correspond approximately to the mean spectrum of the ensemble of $\mathrm{CR}$ sources in the starburst region. ${ }^{43}$

\section{3. $\gamma$-ray Flux Estimate}

If, as argued in the previous section, CR escape from the starburst region is independent of particle energy, the expected total $\gamma$-ray energy flux $F_{\gamma}^{\exp }$ can be estimated simply from the parameters of the system. This independence is approximately true also for the loss rate with respect to inelastic $\mathrm{p}-\mathrm{p}$ collisions; in fact, this loss rate will be approximated by an average over the range of particle energies corresponding to the observed $\gamma$-ray spectrum (see the Appendix). In this case, the particle transport equation can be simply integrated over the energy range of the pion-producing particles. In a leaky-box-type approximation for the starburst region (e.g., Berezinskii et al. 1990), the result can in addition be integrated over the starburst volume. Given that the lifetime of the starburst is about $(2-3) \times 10^{7} \mathrm{yr}$ (see Engelbracht et al. 1998), which is large compared to the advective loss time of about $10^{5} \mathrm{yr}$, the system is in a quasisteady state. This results in the following balance relation for the total non-thermal energy $E_{\mathrm{pp}}^{\pi}$ of pion-producing particles in the starburst region:

$$
E_{\mathrm{pp}}^{\pi}\left[\frac{1}{\tau_{\mathrm{ad}}}+\frac{1}{\tau_{\text {adiab }}}+\frac{1}{\bar{\tau}_{\mathrm{pp}}}\right] \approx Q^{\pi} .
$$

In the derivation of Equation (1) the source spectrum and the resulting spectrum of pion-producing particles in the starburst region are both assumed to have approximately the form of a power-law spectrum in momentum $\propto p^{-\left(\Gamma_{\text {sim }}+2\right)}$, as expected from the theory of diffusive shock acceleration (e.g., Blandford \& Eichler 1987; see the Appendix).

In Equation (1) the quantity $Q^{\pi}=f_{\pi} Q$ is the fraction $f_{\pi}<$ 1 , due to pion-producing particles, of the total input rate $Q$ of non-thermal energy from the $\mathrm{CR}$ sources. Approximately $f_{\pi} \approx$ $3-\Gamma_{\text {sim }}=0.66$, assuming that the observed $\gamma$-ray spectrum with $\Gamma_{\text {sim }}=2.34$ resembles the source spectrum. The quantity $\tau_{\mathrm{ad}}=$ $(H / 2) / v_{\text {wind }} \approx 10^{5} \mathrm{yr}$ denotes the advective loss time, where $v_{\text {wind }} \approx 300 \mathrm{~km} \mathrm{~s}^{-1}$ (Zirakashvili \& Völk 2006) is the velocity of the starburst wind at the top/bottom of the starburst region. The adiabatic loss time is given by $\tau_{\text {adiab }}$ in the accelerating outflow. In a first approximation the flow speed $\boldsymbol{V}$ rises from zero at the galactic symmetry plane in the perpendicular direction $z$ as $|\boldsymbol{V}|=v_{\text {wind }}[|z| /(H / 2)] \mathrm{km} \mathrm{s}^{-1}$, yielding an adiabatic loss rate $1 / \tau_{\text {adiab }} \approx\left(\Gamma_{\text {sim }}-1\right) \nabla \boldsymbol{V} / 3=\left(\Gamma_{\text {sim }}-1\right) /\left(3 \tau_{\text {ad }}\right)$. Finally, $\bar{\tau}_{\mathrm{pp}} \simeq\left(n_{\mathrm{g}} 0.5 c\left\langle\sigma_{\mathrm{pp}}\right\rangle\right)^{-1}$ is an average energy loss time for inelastic, catastrophic proton-proton collisions, which is inversely proportional to the effective gas density $n_{\mathrm{g}}$. The inelasticity is taken as 0.5 . With the mean cross section $\left\langle\sigma_{\mathrm{pp}}\right\rangle \approx$ $33 \mathrm{mb}$ (see the Appendix), this leads to a mean collisional energy loss time $\bar{\tau}_{\mathrm{pp}} \simeq 1.1 \times 10^{5}\left(n_{\mathrm{g}} / 580 \mathrm{~cm}^{-3}\right)^{-1} \mathrm{yr}$.

The total hadronic $\gamma$-ray energy flux density $F_{\gamma}^{\exp }$ and $E_{\mathrm{pp}}^{\pi}$ are connected by

$$
F_{\gamma}^{\exp } \approx \frac{E_{\mathrm{pp}}^{\pi} \eta}{4 \pi d^{2} \bar{\tau}_{\mathrm{pp}}}
$$

\footnotetext{
43 In fact, the $\gamma$-ray spectrum is slightly harder than the parent proton spectrum (Kelner et al. 2006; Kamae et al. 2006; Karlsson 2008).
} 
where $\eta \simeq 1 / 3$ is the $\pi^{0}$-fraction from overall pion production through hadronic collisions.

Equation (2) assumes an optically thin $\gamma$-ray emission region. Indeed, the optical depth $\tau_{\gamma \gamma}$ for $\gamma \gamma$-absorption in the diffuse radiation field in the starburst region and the remaining part of NGC 253 is small compared to unity for the $\gamma$-ray energies considered, i.e., $\tau_{\gamma \gamma}<0.1$ for $\epsilon_{\gamma}<2 \mathrm{TeV}$ (see Inoue 2011).

Assuming that the $\mathrm{CR}$ energy sources in the starburst region are the $\mathrm{SN}$ remnants, then $Q=v_{\mathrm{SN}} \Theta E_{\mathrm{SN}}$, where $\nu_{\mathrm{SN}}$ is the $\mathrm{SN}$ rate, $\Theta<1$ is the $\mathrm{CR}$ production efficiency, and $E_{\mathrm{SN}}$ is the total hydrodynamic energy release per event. In order to comply with the overall energetics of CRs in the Milky Way, an average energy release $\Theta E_{\mathrm{SN}} \approx 10^{50}$ erg into nuclear CRs should be assumed (Drury et al. 1989), with an uncertainty of about a factor of two. For $E_{\mathrm{SN}}=10^{51} \mathrm{erg}$ this implies $\Theta \approx 0.1$.

It is of interest to compare this expected flux with the measured flux $F_{\gamma}^{\text {meas }}(>200 \mathrm{MeV}) \approx 5.3 \times 10^{-12} \mathrm{erg} \mathrm{cm}^{-2} \mathrm{~s}^{-1}$. For this purpose, a nominal value of $0.03 \mathrm{yr}^{-1}$ for $v_{\mathrm{SN}}$ is assumed (Engelbracht et al. 1998). Inserting $E_{\mathrm{pp}}^{\pi}$ from Equation (1) into Equation (2) leads to ${ }^{44}$

$$
\begin{array}{rl}
F_{\gamma}^{\exp } \simeq 2 & 2.6 \times 10^{-11} \mathrm{erg} \mathrm{cm}^{-2} \mathrm{~s}^{-1} \\
\times & {\left[\left(\frac{E_{\mathrm{SN}}}{10^{51} \mathrm{erg}}\right)\left(\frac{\Theta}{0.1}\right)\left(\frac{\nu_{\mathrm{SN}}}{0.03 \mathrm{yr}^{-1}}\right)\left(\frac{f_{\pi}}{0.66}\right)\left(\frac{d}{2.6 \mathrm{Mpc}}\right)^{-2}\right]} \\
\times & {\left[\frac{3}{\left(\bar{\tau}_{\mathrm{pp}} / 1.1 \times 10^{5} \mathrm{yr}\right) /\left(\tau_{\mathrm{ad}} / 10^{5} \mathrm{yr}\right) \times 1.1 \Gamma_{\mathrm{sim}}+3}\right],}
\end{array}
$$

where all parameters like $\left(\tau_{\mathrm{ad}} / 10^{5} \mathrm{yr}\right)$ are written in terms of their nominal values. In the following, the first bracket on the right-hand side of Equation (3) is referred to as the production term and the second bracket is referred to as the loss term.

Considering nominal parameter values in Equation (3), the expected flux $F_{\gamma}^{\exp } \simeq 10^{-11} \mathrm{erg} \mathrm{cm}^{-2} \mathrm{~s}^{-1}$, and then $F_{\gamma}^{\exp } / F_{\gamma}^{\text {meas }} \approx$ 1.9. Given the uncertainties in the measurements of the numerous multi-wavelength parameters involved, the two fluxes are quite close. This supports the general picture on which Equation (3) is based.

The question is to what extent this result leads to physically relevant bounds on physics quantities like the efficiency parameter $\Theta E_{\mathrm{SN}}$. There are two possibilities to reduce the expected $F_{\gamma}^{\exp }$ to the observed value $F_{\gamma}^{\text {meas }}$. The first of them can be achieved by reducing $\Theta E_{\mathrm{SN}}$ compared to its value inferred for the Milky Way and/or by reducing the astrophysical parameter $\nu_{\mathrm{SN}} / d^{2}$, i.e., by reducing the $\mathrm{CR}$ production term in Equation (3). The second possibility is to increase the ratio $\bar{\tau}_{\mathrm{pp}} / \tau_{\mathrm{ad}}$ either by decreasing the effective gas density $n_{\mathrm{g}}$ seen by the pion-producing particles during their advective escape, relative to the average gas density observed, or by increasing $v_{\text {wind }}$. In this second case, the source term could be kept at its nominal value, especially $\Theta E_{\mathrm{SN}}$ could be kept at the value of $10^{50} \mathrm{erg}$. Decreasing $\bar{\tau}_{\mathrm{pp}} / \tau_{\mathrm{ad}}$ relative to its nominal value, on the other hand, is quite implausible for two reasons: first, the wind velocity cannot become significantly smaller without causing difficulties to explain the spatial extent of the starburst region as seen in radio observations (Zirakashvili \& Völk 2006); second, the total gas density cannot significantly increase, given the observations of the molecular gas mentioned earlier. This means that the loss term in Equation (3) has a maximum value for the default parameters in Equation (3). As a

\footnotetext{
44 The assumption that the parent proton spectrum and the resulting $\gamma$-ray spectrum have the same power-law index infers an error of $\approx 20 \%$ in $E_{\mathrm{pp}}^{\pi}$ (see also the Appendix).
}

consequence, the product $\nu_{\mathrm{SN}} \Theta E_{\mathrm{SN}}$ should at most decrease by a factor $1 / 1.9$, if at all, from its value $10^{50} \mathrm{erg} \times 0.03 \mathrm{yr}^{-1}$ for the nominal parameters. Effectively, $\Theta E_{\mathrm{SN}}$ depends on the value $v_{\mathrm{SN}}$. Only for $\nu_{\mathrm{SN}} \gg 0.03 \mathrm{yr}^{-1}$ should $\Theta E_{\mathrm{SN}}$ on average be small compared to $10^{50} \mathrm{erg}$, i.e., in contrast to the average situation in the Milky Way and theoretical expectations for individual SN remnants there.

To derive the quantities of NGC 253, the reference distance of $d=2.6 \mathrm{Mpc}$ has been used most widely in the literature. However, as discussed in the Introduction, this distance has recently been revised to $d=3.5 \mathrm{Mpc}$. Therefore, an appropriate scaling of the astronomical parameters in Equation (3) needs to be considered. Indeed, the total gas mass as determined from the CO line flux scales as $d^{2}$, whereas the starburst volume scales as $d^{3}$. This implies that the gas density scales as $d^{-1}$ and $\bar{\tau}_{\mathrm{pp}} \propto d$. The SN rate is derived from the FIR continuum flux and scales as $v_{\mathrm{SN}} \propto d^{2}$. The wind velocity is derived to be consistent with the geometry of the radio brightness distribution, yielding $v_{\text {wind }} \propto d$. As a consequence, $\tau_{\text {ad }}$ is independent of $d$. For $d=3.5 \mathrm{Mpc}$, the nominal value of $F_{\gamma}^{\exp }$ in Equation (3) is therefore about $8.3 \times 10^{-12} \mathrm{erg} \mathrm{cm}^{-2} \mathrm{~s}^{-1}$. Following the above physical arguments and changing the distance to $d=3.5 \mathrm{Mpc}$, the production term on the right-hand side of Equation (3) must not become smaller by more than a nominal factor $\simeq 1 / 1.6$, in order to reduce $F_{\gamma}^{\exp }$ to $F_{\gamma}^{\text {meas }}$.

In general, the simple model presented here agrees quite well with the observed values, given the observational uncertainties of the astronomical parameters and the possibility that for SN remnants in such a dense medium the total non-thermal energy generated per event $\Theta E_{\mathrm{SN}}$ might indeed be lower by a factor $\simeq 1 / 1.6$ than typically assumed for an object in the average ISM of the Milky Way.

\subsection{Hadronic Calorimetry}

In this scenario, to find the extent to which the starburst region behaves calorimetrically in the presence of advective and diffusive escape, the total energy production rate in hadronic collisions $L_{\text {coll }}=4 \pi d^{2} F_{\gamma}^{\text {meas }} / \eta$ is compared with the total production $L_{\mathrm{CR}}(\pi)=f_{\pi} v_{\mathrm{SN}} \Theta E_{\mathrm{SN}}$ of CRs capable of producing hadronic $\gamma$-rays. Such a comparison for NGC 253 has previously been made by Aharonian et al. (2005), Loeb \& Waxman (2006), and Thompson et al. (2007). $L_{\mathrm{CR}}(\pi)$ depends on the fraction of energy available for pion production $f_{\pi} \approx 3-s$, with $s$ being the source spectral index. In the present scenario $f_{\pi} \simeq \Gamma_{\text {sim }}$ and therefore $f_{\pi}=0.66$.

Adopting a value of $0.03 \mathrm{yr}^{-1}$ for $v_{\mathrm{SN}}$ (Engelbracht et al. 1998) and assuming $\Theta E_{\mathrm{SN}} \approx 10^{50} \mathrm{erg}$ again results in

$$
\begin{aligned}
\frac{L_{\mathrm{coll}}}{L_{\mathrm{CR}}(\pi)} \approx & 0.21 \times\left(\frac{F_{\gamma}^{\text {meas }}}{5.3 \times 10^{-12} \mathrm{erg} \mathrm{cm}^{-2} \mathrm{~s}^{-1}}\right) \\
\times & {\left[\left(\frac{E_{\mathrm{SN}}}{10^{51} \mathrm{erg}}\right)\left(\frac{\Theta}{0.1}\right)\left(\frac{\nu_{\mathrm{SN}}}{0.03 \mathrm{yr}^{-1}}\right)\left(\frac{f_{\pi}}{0.66}\right)\left(\frac{d}{2.6 \mathrm{Mpc}}\right)^{-2}\right]^{-1} . }
\end{aligned}
$$

This fraction of about $20 \%$ is then a measure of the extent to which the starburst region is calorimetric with respect to its hadronic interactions, dissipating its own non-thermal output; and since $v_{\mathrm{SN}}$ is proportional to the FIR luminosity and hence to $d^{2}$, this result is formally independent of the distance $d$. The ratio $L_{\mathrm{coll}} / L_{\mathrm{CR}}(\pi)$ is comparable to the value found by Lacki et al. (2011). Like $F_{\gamma}^{\exp } / F_{\gamma}^{\text {meas }}$, it is affected by the uncertainties 
of the input parameters. Adopting a larger ratio of $v_{\mathrm{SN}} / d^{2}$ would decrease this value, whereas a lower efficiency $\Theta E_{\mathrm{SN}}$ would increase it. The difference between $F_{\gamma}^{\exp }$ and $F_{\gamma}^{\text {meas }}$, discussed above, shows that the value of the production term of Equation (3) should possibly be smaller than unity but not smaller than $\simeq 1 / 1.6$ for $d=3.5 \mathrm{Mpc}$. This suggests that the calorimetric fraction may be larger than $20 \%$, possibly reaching up to about $30 \%$.

\subsection{Cosmic-ray Energy Density and Magnetic Field Strength}

Within this framework the non-thermal energy density $U_{\mathrm{pp}}^{\pi}$ of the $\pi^{0}$-producing particles in the starburst region can be simply calculated from Equation (2) by substituting $F_{\gamma}^{\exp }=F_{\gamma}^{\text {meas }}$ and dividing the resulting $E_{\mathrm{pp}}^{\pi}$ by the estimated volume. This gives $U_{\mathrm{pp}} \approx 230 \mathrm{eV} \mathrm{cm}^{-3}$, independent of $d$. This is a lower limit for the total non-thermal energy density $U_{\mathrm{pp}}$. Since it is difficult to estimate the contribution of the lower-energy particles produced in the sources, because of their poorly determined ionization losses in the outflow, one can only give an upper limit $U_{\mathrm{pp}}<U_{\mathrm{pp}}^{\pi} / f_{\pi} \approx 340 \mathrm{eV} \mathrm{cm}^{-3}$. The values in this range are more than a hundred times larger than the CR energy density in the Milky Way and, by implication, on average in the disk of NGC 253 (see the Introduction). Assuming, as for the Milky Way, a CR scale length of about $1 \mathrm{kpc}$ for the extended disk of NGC 253 in comparison to the $\approx 40 \mathrm{pc}$ gradient scale in the starburst region (for $d=3.5 \mathrm{Mpc}$ ), the latter's CR pressure gradient is almost four orders of magnitude larger than that on average in the disk.

Equating $U_{\mathrm{pp}}$ with the magnetic field energy density $\boldsymbol{B}^{2} / 8 \pi$ in the sense of a conventional equipartition argument for magnetic field and CRs results in a range for the rms magnetic field strength $90 \mu \mathrm{G} \lesssim B_{\text {eq }} \lesssim 120 \mu \mathrm{G}$. Note that the estimates of $U_{\mathrm{pp}}$ and $B_{\mathrm{eq}}$ are also affected by the uncertainty of the inelasticity factor for $\mathrm{p}-\mathrm{p}$ collisions that could be $\approx 20 \%$ lower (Musulmanbekov 2004). This is to be compared with the $(160 \pm 20) \mu \mathrm{G}$ equipartition field strength for the so-called nuclear region, estimated recently by Heesen et al. (2011) from radio continuum observations and assuming a proton-toelectron ratio of 100 .

\subsection{Contribution of Discrete Sources}

It has recently been suggested that the $\mathrm{HE} \gamma$-ray emission from NGC 253 might indeed come from interactions of diffuse nuclear CRs with the ambient gas, but that this contribution should substantially diminish at energies $\geqslant 10 \mathrm{GeV}$ as a result of energy-dependent diffusion losses from the starburst region (Mannheim et al. 2012). The observed TeV emission was argued to be due to inverse Compton emission from unresolved pulsar wind nebulae (PWNe) instead, i.e., from a separate population of discrete sources. For such PWNe, the evolution was assumed to be the same in the disk of the Milky Way and in the extremely high-density environment of the starburst nucleus of NGC 253. It has to be noted, however, that electron cooling times in typical starburst environments are very short and electrons are expected to cool on timescales of a few hundred years (Ohm \& Hinton 2012).

While in the present paper it is argued that advective removal should dominate over diffusive losses of energetic nuclear particles deep into the $\mathrm{TeV}$ range, a discussion of the role of PWNe in such an environment is beyond the scope of the present work. On the other hand, the observations show that the spectrum of NGC 253 can be well described by a single power law over the combined HE and VHE energy range, disfavoring two distinct spectral components and suggesting that the same physical processes dominate over the entire energy range in question. Therefore, additional discrete $\gamma$-ray sources seem to play a minor role.

\section{SUMMARY AND OUTLOOK}

The analysis results of $177 \mathrm{hr}$ of H.E.S.S. data obtained in observations of the starburst galaxy NGC 253 are reported. The reconstructed energy spectrum is best described by a power law with differential photon index $\Gamma=2.14 \pm 0.18_{\text {stat }} \pm 0.30_{\text {sys }}$ and differential flux normalization at $1 \mathrm{TeV}$ of $F_{0}=(9.6 \pm$ $\left.1.5_{\text {stat }}(+5.7,-2.9)_{\text {sys }}\right) \times 10^{-14} \mathrm{TeV}^{-1} \mathrm{~cm}^{-2} \mathrm{~s}^{-1}$. In addition to the H.E.S.S. data, the analysis of the 30 month Fermi-LAT data set revealed an improved best-fit position compatible with the optical center of the galaxy and with the H.E.S.S. source within statistical errors. The reconstructed differential photon index is $\Gamma=2.24 \pm 0.14_{\text {stat }} \pm 0.03_{\text {sys }}$, and the integral flux between 0.2 and $200 \mathrm{GeV}$ is $F(0.2-200 \mathrm{GeV})=\left(4.9 \pm 1.0_{\text {stat }} \pm\right.$ $\left.0.3_{\text {sys }}\right) \times 10^{-9} \mathrm{~cm}^{-2} \mathrm{~s}^{-1}$. The HE and VHE $\gamma$-ray spectra of the starburst region can be described by a simultaneous powerlaw fit with differential photon index $\Gamma_{\text {sim }}=2.34 \pm 0.03$ and a fit probability of $30 \%$. This result implies that no spectral break or turnover is required to explain the $\gamma$-ray data. The corresponding total energy flux density corresponds to $F^{E}(>200 \mathrm{MeV}) \approx 5.3 \times 10^{-12} \mathrm{erg} \mathrm{cm}^{-2} \mathrm{~s}^{-1}$. Assuming the remaining disk of NGC 253 to be quantitatively similar to the Milky Way, the starburst region outshines the rest of NGC 253 by an order of magnitude in HE and VHE $\gamma$-rays, consistent with the detection of the object as an H.E.S.S. point source.

Model predictions that assume a dominantly hadronic origin of the $\gamma$-ray emission are roughly compatible with the spectral results presented in this work. For a set of reasonable parameters the CR energy, which is lost in $\mathrm{p}-\mathrm{p}$ interactions and partly reappears in $\pi^{0}$-decay $\gamma$-ray production, is inferred in the present work as $\approx 20 \%$ and possibly up to $\approx 30 \%$ of the total non-thermal energy produced in the starburst region, assuming a distance of 3.5 Mpc and a $10 \%$ efficiency for CR acceleration in starburst SN remnants. Note, however, that the multi-wavelength observables are only known within a considerable error margin, which can change these percentages significantly. CRs are also removed by diffusion and advection from the starburst region. Since the former process is energy dependent, a spectral steepening with energy would be expected. The smooth alignment of the HE and VHE $\gamma$-ray spectra over four decades in energy hence indicates that advective losses in NGC 253 most likely dominate from a few $\mathrm{GeV}$ to more than $10 \mathrm{TeV}$. Even at such HEs the diffusion coefficient would still be more than two orders of magnitude larger than the Bohm diffusion coefficient. It therefore seems likely that the observed spectrum can be characterized by the same photon index as the average particle accelerator in the starburst region.

The form of the $\gamma$-ray spectrum of NGC 253 can be compared with another starburst galaxy, M82 in the Northern Hemisphere, detected by the Fermi-LAT (Abdo et al. 2010a) and VERITAS (Acciari et al. 2009) Collaborations, respectively. If one looks at the corresponding $\mathrm{HE}$ and VHE $\gamma$-ray spectra of M82, the overall shape looks rather similar to the one presented in this paper for NGC 253. Even though the starburst in M82 is in all probability triggered by the interaction with the companion galaxy M81, the spectral similarity is consistent with the assumption that the $\mathrm{CR}$ sources in both galaxies produce similar 
energetic particle spectra and may therefore be of the same nature.

We thank S. R. Kelner for valuable discussions and the anonymous referee for his very useful comments. The support of the Namibian authorities and of the University of Namibia in facilitating the construction and operation of H.E.S.S. is gratefully acknowledged, as is the support by the German Ministry for Education and Research (BMBF), the Max Planck Society, the German Research Foundation (DFG), the French Ministry for Research, the CNRS-IN2P3 and the Astroparticle Interdisciplinary Programme of the CNRS, the U.K. Science and Technology Facilities Council (STFC), the IPNP of the Charles University, the Czech Science Foundation, the Polish Ministry of Science and Higher Education, the South African Department of Science and Technology and National Research Foundation, and the University of Namibia. We appreciate the excellent work of the technical support staff in Berlin, Durham, Hamburg, Heidelberg, Palaiseau, Paris, Saclay, and Namibia in the construction and operation of the equipment. S.O. acknowledges the support of the Humboldt foundation by a Feodor-Lynen research fellowship.

\section{APPENDIX}

Equation (1) of the main text can be derived from the transport equation for the isotropic part $f(\boldsymbol{x}, p, t)$ of the particle momentum distribution in a simple model, neglecting the speed of the scattering fluctuations compared to the fluid mass velocity $\boldsymbol{V}$ as well as diffusion in momentum space (e.g., Blandford \& Eichler 1987):

$$
\begin{aligned}
\tilde{Q}(\boldsymbol{x}, p, t)= & \frac{\partial f}{\partial t}-\nabla(\kappa \nabla f)+\nabla(\boldsymbol{V} f) \\
& -\frac{1}{p^{2}} \frac{\partial}{\partial p}\left(p^{2} \frac{p}{3}(\nabla \boldsymbol{V}) f\right)-\frac{f}{\bar{\tau}_{\mathrm{pp}}},
\end{aligned}
$$

where $\kappa(p)$ denotes the spatial diffusion coefficient, $\bar{\tau}_{\mathrm{pp}}$ is the average energy loss time as a result of inelastic, catastrophic nuclear collisions (assumed to be energy independent for $p>$ $2 m_{\mathrm{p}} c$ and infinite for $p<2 m_{\mathrm{p}} c$; see below), and $\tilde{Q}(\boldsymbol{x}, p, t)$ is the particle production rate of the $\mathrm{CR}$ sources. The adiabatic loss rate of a particle in the accelerating flow is given by $p / 3 \nabla \boldsymbol{V}$. In the energy range of pion-producing nuclei ionization losses are neglected. Assuming a steady state $(\partial f / \partial t=0)$ and neglecting particle diffusion $(\kappa=0)$, the flow velocity $\boldsymbol{V}$ is approximated to be perpendicular to the galactic disk mid-plane, varying as $\boldsymbol{V}=\left(0,0, v_{\text {wind }} \times z(H / 2)^{-1}\right)$ for $0<|z|<H / 2$, where the constant parameter $v_{\text {wind }}$ is the wind velocity at the starburst boundary.

To obtain the balance relation for the kinetic energy of the pion-producing CRs $E_{\mathrm{pp}}^{\pi}=\int_{V_{\mathrm{SB}}} d^{3} x 4 \pi \int_{p_{\min }}^{p_{\max }} d p p^{2} E_{\mathrm{kin}} f$ in the starburst region, Equation (A1) is multiplied by the particle kinetic energy $E_{\text {kin }}$ and integrated over the relevant momenta as well as over the spatial starburst volume $V_{\mathrm{SB}}$. In a leaky box approximation for the starburst region (e.g., Berezinskii et al. 1990), $f(\boldsymbol{x}, p)$ and $\bar{\tau}_{\mathrm{pp}}$ are assumed to be spatially uniform. It is then clear for this particle distribution that it has the same momentum dependence as the spatially averaged source production rate $\int_{V_{\mathrm{SB}}} d^{3} x \tilde{Q}(\boldsymbol{x}, p) / V_{\mathrm{SB}}$ for $p_{\min }<p<p_{\max }$. In addition, it is assumed that the production spectrum in the sources is not strongly influenced by energy-dependent losses in the sources themselves. The averaged particle source production rate can then be assumed to have a power-law dependence $\propto p^{-(s+2)}$ in particle momentum $p$ for all momenta above the injection energy, consistent with an origin of the CR particles from diffusive shock acceleration (e.g., Blandford \& Eichler 1987). And $s \approx \Gamma_{\text {sim }}$, where $\Gamma_{\text {sim }}=2.34$ is the power-law index of the observed $\gamma$-ray flux. The minimum momentum $p_{\min }$ in Equation (A1) roughly corresponds to $\mathrm{GeV}$ energies. Here $p_{\text {min }}$ is taken as $2 m_{\mathrm{p}} c$. Since the maximum observed $\gamma$-ray energy is $4.7 \mathrm{TeV}$, corresponding to a proton energy of $c p_{\max } \approx 30 \mathrm{TeV}$, momenta $p>p_{\max }$ make a negligible contribution. Therefore, it is possible to put $p_{\max }=\infty$.

In order to obtain an analytical estimate, the above integral for $E_{\mathrm{pp}}^{\pi}$ is approximated by using the relativistic formula $E_{\mathrm{kin}}=c p$, for $p>2 m_{\mathrm{p}} c$ (Drury et al. 1989).

Integrating the individual terms in Equation (A1) by parts over momentum results in

$$
E_{\mathrm{pp}}^{\pi}\left[\frac{1}{\tau_{\mathrm{ad}}}+\frac{1}{\tau_{\mathrm{adiab}}}+\frac{1}{\bar{\tau}_{\mathrm{pp}}}\right] \approx Q^{\pi},
$$

where $\tau_{\text {ad }}=(H / 2) / v_{\text {wind }}, \tau_{\text {adiab }} \approx 3 \tau_{\text {ad }} /\left(\Gamma_{\text {sim }}-1\right)$, and $Q^{\pi} \approx 4 \pi \int_{2 m_{\mathrm{p}} c}^{\infty} d p p^{2} \tilde{Q}$. This quantity $Q^{\pi}=f_{\pi} Q$ defines the fraction $f_{\pi}<1$ of the total particle energy production $Q=\int_{V_{\mathrm{SB}}} d^{3} x 4 \pi \int_{0}^{\infty} d p p^{2} E_{\mathrm{kin}} \tilde{Q}$, integrated over all momenta, that is available for pion production. Consistent with the approximation for $Q^{\pi}$, the integral for $Q$ is approximated by using the Newtonian formula $E_{\mathrm{kin}}=p^{2} / 2 m_{\mathrm{p}}$ for $p<2 m_{\mathrm{p}} c$ and the relativistic formula $E_{\mathrm{kin}}=c p$ for $p>2 m_{\mathrm{p}} c$ (Drury et al. 1989).

Assuming $2 \lesssim s \lesssim 3, p_{\text {inj }} \ll 2 m_{\mathrm{p}} c$, for the injection momentum $p_{\text {inj }}$, and $p_{\max } \gg 2 m_{\mathrm{p}} c$ then gives

$$
\begin{aligned}
Q^{\pi} & \approx 4 \pi Q_{0} \int_{2 m_{\mathrm{p}} c}^{p_{\max }} d p p^{2} c p\left(p / 2 m_{\mathrm{p}} c\right)^{-s-2} \\
& \approx 4 \pi Q_{0} c /(s-2)\left(2 m_{\mathrm{p}} c\right)^{4}
\end{aligned}
$$

and

$$
\begin{aligned}
Q-Q^{\pi} & \approx 4 \pi Q_{0} \int_{p_{\min }}^{2 m_{\mathrm{p}} c} d p p^{2} p^{2} / 2 m_{\mathrm{p}}\left(p / 2 m_{\mathrm{p}} c\right)^{-s-2} \\
& \approx 4 \pi Q_{0} c /(3-s)\left(2 m_{\mathrm{p}} c\right)^{4} .
\end{aligned}
$$

Therefore, $Q^{\pi} \approx(3-s) Q$ and $f_{\pi} \approx 3-s$ for the assumed momentum spectrum. For $s=\Gamma_{\operatorname{sim}}=2.34, f_{\pi} \approx 0.66$. For a very hard source spectrum with $s$ close to $2, f_{\pi}$ would be close to 1. Equation (A2) corresponds to Equation (1) of the main text.

The approximate quantity $\bar{\tau}_{\mathrm{pp}}$, i.e., the mean loss time that appears in Equation (A1), arises from averaging the energy loss rate $\tau_{\mathrm{pp}}^{-1}(E)$ over the differential energy spectrum of the colliding particles. Approximating the differential energy dependence of the particle spectrum by that of the observed $\gamma$-ray spectrum implies that the average can be taken over a power-law spectrum $\tilde{f}\left(E_{\text {kin }}\right) \propto E_{\text {kin }}^{-\Gamma_{\text {sim }}}$, which has the same index $\Gamma_{\text {sim }}=2.34$ as the differential $\gamma$-ray flux as a function of the $\gamma$-ray energy $\epsilon_{\gamma}$ in the energy region observed for NGC 253, thus defining

$$
\frac{1}{\bar{\tau}_{\mathrm{pp}}}=\frac{\int d E E \tilde{f}(E) / \tau_{\mathrm{pp}}}{\int d E E \tilde{f}(E)} .
$$

The integration limits are the particle (proton) energies corresponding to the observed energy range of the $\gamma$-ray energy spectrum. Since $\tau_{\mathrm{pp}}^{-1}=n_{\mathrm{g}} c f_{\mathrm{in}} \sigma_{\mathrm{pp}}\left(E_{\mathrm{kin}}\right)$ with the total cross section 
$\sigma_{\mathrm{pp}}$ for inelastic pp collisions and inelasticity factor $f_{\text {in }} \approx 0.5$ (see Aharonian 2004), this amounts to the following average over the inelastic nuclear cross-section $\sigma_{\mathrm{pp}}$ :

$$
\frac{1}{\bar{\tau}_{\mathrm{pp}}}=\frac{n_{\mathrm{g}} c f_{\mathrm{in}} \int d E E \sigma_{\mathrm{pp}}(E) \tilde{f}(E)}{\int d E E \tilde{f}(E)} .
$$

It is a convenient approximation to choose the integration limits as $E_{\mathrm{kin}}^{\min }=1 \mathrm{GeV}$, and as $E_{\mathrm{kin}}^{\max } \approx(4.7 \mathrm{TeV} / 0.17) \approx 30 \mathrm{TeV}$ according to the maximum $\gamma$-ray energy $\epsilon_{\gamma}=4.7 \mathrm{TeV}$ observed. For $E_{\text {kin }}>1 \mathrm{GeV}$, the cross section $\sigma_{\mathrm{pp}}$ is approximated in the following form (Aharonian 2004):

$$
\sigma_{\mathrm{pp}}\left(E_{\mathrm{kin}}\right) \approx 30\left[0.95+0.06 \ln \left(E_{\mathrm{kin}} / \mathrm{GeV}\right)\right] \mathrm{mb}
$$

The corresponding integrals can be calculated analytically. Since the spectrum is relatively soft, the resulting effective cross section $\left\langle\sigma_{\mathrm{pp}}\right\rangle \approx 33 \mathrm{mb}$ corresponds to a rather low effective particle energy $\left\langle E_{\text {kin }}\right\rangle \approx 12 \mathrm{GeV}$. Due to the slightly softer source spectral index compared to the resulting $\gamma$-ray spectral index $\left(s \simeq 2.4\right.$ versus $\Gamma_{\text {sim }}=2.34$ ), the assumption of equal spectral indices infers an error of $\approx 20 \%$ of the proton energy density $E_{\mathrm{pp}}^{\pi}$ in Equations (3) and (4) of the main text (S. R. Kelner 2012, private communication). This is certainly a negligible effect, compared to all other uncertainties.

\section{REFERENCES}

Abdo, A. A., Ackermann, M., Ajello, M., et al. (Fermi-LAT Collaboration) 2009, ApJ, 707, 1310

Abdo, A. A., Ackermann, M., Ajello, M., et al. (Fermi-LAT Collaboration) 2010a, ApJ, 709, L152

Abdo, A. A., Ackermann, M., Ajello, M., et al. (Fermi-LAT Collaboration) 2010b, ApJS, 188, 405

Acciari, V. A., Aliu, E., Arlen, T., et al. (VERITAS Collaboration) 2009, Nature, 462,770

Acero, F., Aharonian, F., Akhperjanian, A. G., et al. (H.E.S.S. Collaboration) 2009, Science, 326, 1080

Aharonian, F., Akhperjanian, A. G., Bazer-Bachi, A. R., et al. (H.E.S.S. Collaboration) 2005, A\&A, 442, 177

Aharonian, F., Akhperjanian, A. G., Bazer-Bachi, A. R., et al. (H.E.S.S. Collaboration) 2006, A\&A, 457, 899

Aharonian, F. A. 2004, Very High Energy Cosmic Gamma Radiation: A Crucial Window on the Extreme Universe (1st ed.; Singapore: World Scientific)

Akyuz, A., Brouillet, N., \& Ozel, M. E. 1991, A\&A, 248, 419

Atwood, W. B., Abdo, A. A., Ackermann, M., et al. (Fermi-LAT Collaboration) 2009, ApJ, 697, 1071

Berezinskii, V. S., Bulanov, S. V., Dogiel, V. A., \& Ptuskin, V. S. (ed.) 1990 Astrophysics of Cosmic Rays (Amsterdam: Elsevier)

Berge, D., Funk, S., \& Hinton, J. 2007, A\&A, 466, 1219

Blandford, R., \& Eichler, D. 1987, Phys. Rep., 154, 1

Breitschwerdt, D., McKenzie, J. F., \& Völk, H. J. 1991, A\&A, 245, 79

Bykov, A. M. 2001, Space Sci. Rev., 99, 317

Dalcanton, J. J., Williams, B. F., Seth, A. C., et al. 2009, ApJS, 183, 67
Davidge, T. J., Le Fevre, O., \& Clark, C. C. 1991, ApJ, 370, 559

de Naurois, M., \& Rolland, L. 2009, Astropart. Phys., 32, 231

Domingo-Santamaría, E., \& Torres, D. F. 2005, A\&A, 444, 403

Drury, L. O., Markiewicz, W. J., \& Völk, H. J. 1989, A\&A, 225, 179

Engelbracht, C. W., Rieke, M. J., Rieke, G. H., Kelly, D. M., \& Achtermann, J. M. 1998, ApJ, 505, 639

Feldman, G. J., \& Cousins, R. D. 1998, Phys. Rev. D, 57, 3873

Heesen, V., Beck, R., Krause, M., \& Dettmar, R.-J. 2009, A\&A, 494, 563

Heesen, V., Beck, R., Krause, M., \& Dettmar, R.-J. 2011, A\&A, 535, A79

Hillas, A. M. 1985, in 19th Intern. Cosmic Ray Conf., Vol. 3, ed. F. C. Jones, 445

Inoue, Y. 2011, ApJ, 728, 11

Kamae, T., Karlsson, N., Mizuno, T., Abe, T., \& Koi, T. 2006, ApJ, 647, 692

Karachentsev, I. D., Grebel, E. K., Sharina, M. E., et al. 2003, A\&A, 404, 93

Karlsson, N. 2008, in AIP Conf. Ser. 1085, High Energy Gamma-ray Astronomy, ed. F. A. Aharonian, W. Hofmann, \& F. Rieger (Melville, NY: AIP), 561

Kelner, S. R., Aharonian, F. A., \& Bugayov, V. V. 2006, Phys. Rev. D, 74, 034018

Lacki, B. C., Thompson, T. A., \& Quataert, E. 2010, ApJ, 717, 1

Lacki, B. C., Thompson, T. A., Quataert, E., Loeb, A., \& Waxman, E. 2011, ApJ, 734, 107

Li, T., \& Ma, Y. 1983, ApJ, 272, 317

Loeb, A., \& Waxman, E. 2006, J. Cosmol. Astropart. Phys., JCAP05(2006)003

Mannheim, K., Elsässer, D., \& Tibolla, O. 2012, Astropart. Phys., 35, 797

Mattox, J. R., Bertsch, D. L., Chiang, J., et al. 1996, ApJ, 461, 396

Mauersberger, R., Henkel, C., Wielebinski, R., Wiklind, T., \& Reuter, H.-P. 1996, A\&A, 305, 421

Melo, V. P., Pérez García, A. M., Acosta-Pulido, J. A., Muñoz-Tuñón, C., \& Rodríguez Espinosa, J. M. 2002, ApJ, 574, 709

Musulmanbekov, G. 2004, Phys. At. Nucl., 67, 90

Nolan, P. L., Abdo, A. A., Ackermann, M., et al. (Fermi-LAT Collaboration) 2012, ApJS, 199, 31

Ohm, S., \& Hinton, J. A. 2012, in IAU Symp. 284, The Spectral Energy Distribution of Galaxies, ed. R. J. Tuffs \& C. C. Popescu (Cambridge: Cambridge Univ. Press), 382

Ohm, S., van Eldik, C., \& Egberts, K. 2009, Astropart. Phys., 31, 383

Paglione, T. A. D., Marscher, A. P., Jackson, J. M., \& Bertsch, D. L. 1996, ApJ, 460, 295

Paglione, T. A. D., Tosaki, T., \& Jackson, J. M. 1995, ApJ, 454, L117

Parizot, E., Marcowith, A., van der Swaluw, E., Bykov, A. M., \& Tatischeff, V. 2004, A\&A, 424, 747

Pence, W. D. 1980, ApJ, 239, 54

Ptuskin, V. S., Völk, H. J., Zirakashvili, V. N., \& Breitschwerdt, D. 1997, A\&A, 321,434

Rekola, R., Richer, M. G., McCall, M. L., et al. 2005, MNRAS, 361, 330

Rephaeli, Y., Arieli, Y., \& Persic, M. 2010, MNRAS, 401, 473

Sakamoto, K., Mao, R.-Q., Matsushita, S., et al. 2011, ApJ, 735, 19

Sorai, K., Nakai, N., Kuno, N., Nishiyama, K., \& Hasegawa, T. 2000, PASJ, 52, 785

Strong, A. W., Moskalenko, I. V., \& Ptuskin, V. S. 2007, Ann. Rev. Nucl. Part. Sci., 57, 285

Strong, A. W., Porter, T. A., Digel, S. W., et al. 2010, ApJ, 722, L58

Thompson, T. A., Quataert, E., \& Waxman, E. 2007, ApJ, 654, 219

Van Buren, D., \& Greenhouse, M. A. 1994, ApJ, 431, 640

Völk, H. J., Aharonian, F. A., \& Breitschwerdt, D. 1996, Space Sci. Rev., 75, 279

Völk, H. J., Klein, U., \& Wielebinski, R. 1989, A\&A, 213, L12

Weaver, K. A., Heckman, T. M., Strickland, D. K., \& Dahlem, M. 2002, ApJ, 576, L19

Zirakashvili, V. N., \& Völk, H. J. 2006, ApJ, 636, 140 